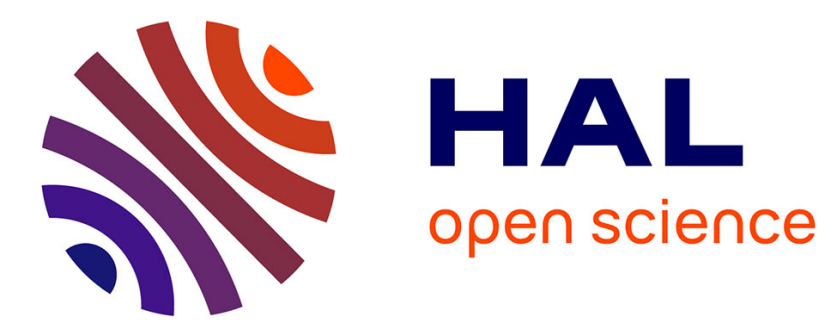

\title{
Theory of Ultrafast Photoinduced Heterogeneous Electron Transfer
}

Volkhard May

\section{To cite this version:}

Volkhard May. Theory of Ultrafast Photoinduced Heterogeneous Electron Transfer. Molecular Simulation, 2006, 32 (09), pp.765-781. 10.1080/08927020600883285 . hal-00514996

\section{HAL Id: hal-00514996 \\ https://hal.science/hal-00514996}

Submitted on 4 Sep 2010

HAL is a multi-disciplinary open access archive for the deposit and dissemination of scientific research documents, whether they are published or not. The documents may come from teaching and research institutions in France or abroad, or from public or private research centers.
L'archive ouverte pluridisciplinaire HAL, est destinée au dépôt et à la diffusion de documents scientifiques de niveau recherche, publiés ou non, émanant des établissements d'enseignement et de recherche français ou étrangers, des laboratoires publics ou privés. 


\section{Molecular Simulation}

Journal of

Experimental Nanoscience

Taylor \& Francis

Taglor \& Francis Group

\section{Theory of Ultrafast Photoinduced Heterogeneous Electron Transfer}

\begin{tabular}{|r|l|}
\hline Journal: & Molecular Simulation/Journal of Experimental Nanoscience \\
\hline Manuscript ID: & GMOS-2006-0052.R1 \\
\hline Journal: & Molecular Simulation \\
\hline Date Submitted by the \\
Author: & 27-Jun-2006 \\
\hline Complete List of Authors: & May, Volkhard; Humboldt-University at Berlin, Institute of Physics \\
\hline Keywords: & $\begin{array}{l}\text { ultrafast heterogeneous electron transfer, perylene on TiO_2, } \\
\text { molecular Green's function }\end{array}$ \\
\hline
\end{tabular}

\section{SCHOLARONE ${ }^{m}$ Manuscripts}




\title{
Theory of Ultrafast Photoinduced Heterogeneous Electron Transfer
}

\author{
Luxia Wang, ${ }^{1}$ Frank Willig, ${ }^{2}$ and Volkhard May ${ }^{1}$ \\ ${ }^{1}$ Institut für Physik, Humboldt-Universität zu Berlin, \\ Newtonstraße 15, D-12489 Berlin, Germany \\ ${ }^{2} H a h n-M e i t n e r-I n s t i t u t$, Abteilung Dynamik von Genzflächenreaktionen \\ Glienicker Str. 100, 14109 Berlin, Germany
}

(Dated: June 27, 2006) 


\begin{abstract}
Ultrafast heterogeneous electron transfer (HET) between a molecule attached to a semiconductor surface and the conduction band of the semiconductor is discussed theoretically with emphasis on the perylene $\mathrm{TiO}_{2}$ system. The used description accounts for the specialty of the molecule i.e. its particular electronic level scheme together with its vibrational degrees of freedom. The band continuum of the semiconductor is included and the approach is ready to describe different optical excitation and detection processes. Using a diabatic-state like separation of the whole system into molecular and semiconductor states femtosecond photoinduced dynamics are studied. Since the HET is ultrafast standard rate theories cannot be applied. Instead, the respective time-dependent Schrödinger equation governing the electron-vibrational wave function is solved. Based on this approach and using a time-dependent formulation the steady state linear absorption is calculated. Parameters of perylene attached to nano-structured $\mathrm{TiO}_{2}$ via different bridge-anchor groups are adjusted by a comparison with measured spectra. A direct charge transfer excitation into the conduction band continuum is included into the description. This time-dependent formulation of the absorbance is confronted with a direct formulation in the frequency domain using the molecular Green's function. Then, it is explained how to observe the energetic distribution of the injected electron which carries signatures of the molecular vibrations in a two-photon photon emission spectrum. Some speculations on a laser pulse control of ultrafast HET are finally given.
\end{abstract}

\title{
Keywords:
}

ultrafast heterogeneous electron transfer, perylene on $\mathrm{TiO}_{2}$, steady state linear absorption, molecular Green's function, two-photon photon emission spectrum, laser pulse control, 


\section{INTRODUCTION}

Electron transfer (ET) represents a ubiquitous phenomenon in physics, chemistry, and biology which attracted continuous interest over the last decades (see this volume and $[1-5])$. With the dawn of femtosecond spectroscopy activities have also been directed to sub-picosecond transfer processes. Although some experimental data are available on subpicosecond photoinduced ET the field is dominated by theoretical activities focusing on wave packet dynamics in donor-acceptor complexes beyond the nonadiabatic transfer. If the optical preparation is fast enough one may follow the formation of a vibrational wave packet in the donor state and its motion into the acceptor state. This process has been studied under various respects, related, e.g., to the importance of dissipative effects in the course of the wavepacket motion, to the influence of electronic and vibrational dephasing, to the type of optical excitation etc. (cf. [6-8] for the early contributions and, e.g., Refs. [9-13] for some recent activities).

Since experimental examples on ultrafast donor-acceptor ET are rather rare, femtosecond interfacial heterogeneous electron transfer (HET) became of great interest during recent years. Different molecule sensitized semiconductor surfaces have been investigated, and it has been reported on HET proceeding on a sub $100 \mathrm{fs}$ and even on a sub $10 \mathrm{fs}$ time region. Although there are practical device applications of HET reactions, e.g. in nano-hybrid systems [14-19], the exploration of these transfer processes is an interesting topic in its own rights (see, for example, [20-22]).

Perylene attached to nano-structured $\mathrm{TiO}_{2}$ is of particular interest in this connection. It is well suited for a systematic study of photoinduced ultrafast HET since the first excited electronic state of perylene is energetically positioned about $1 \mathrm{eV}$ above the conduction band edge of $\mathrm{TiO}_{2}$ thus realizing a mid-band charge injection situation. Introducing different bridge-anchor groups the transfer coupling which initiates HET can be tuned from a strongcoupling situation (with charge injection times of $10 \mathrm{fs}$ ) down to weaker coupling strengths (with charge injection times of up to 1 ps [23]).

The present paper continues our theoretical studies on HET with particular emphasis on the perylene- $\mathrm{TiO}_{2}$ system [24-29]. To account for the optical excitation, for subsequent electron-vibrational quantum dynamics, the formation of an electron distribution in the semiconductor band continuum, its detection by two-photon photon emission spec- 
troscopy, and for femtosecond laser pulse control a model of reduced dimensionality has to be applied. This reduction concerns the electronic levels involved as well as the number of intra-molecular vibrational coordinates. Similar computations have been carried out in Refs. $[30,31]$ but with a more involved account on vibrational dynamics dropping at the same time any consideration on the state of the injected electron in the conduction band continuum. Such considerations, however, have been in the focus of DFT-based electron structure calculation of dye sensitized semiconductor surfaces given, for example, in Refs. [32-35]. In particular, a combination of electronic structure calculations with molecular dynamics simulations have been presented in [33, 34]. So far, however, these sophisticated computations could not be directed up to measured data obtained, for example, in a femtosecond optical experiment.

Since the initiation and detection of the type of HET discussed here is based on ultrafast optical techniques a certain part of the presentation focuses on the computation of optical spectra. After some qualitative discussion in the subsequent section the general HET model is introduced in Section III with a specification in Section III A to the simple charge transfer model used for the perylene- $\mathrm{TiO}_{2}$ system so far. The way to simulate femtosecond charge injection dynamics is explained in Section IV and illustrated with some results valid for perylene on $\mathrm{TiO}_{2}$. In Section $\mathrm{V}$ we demonstrate the computation of the linear absorption coefficient which is of central importance for parameter adjustment. Preliminary data on two-photon photon emission spectra are presented afterwards. The discussion is finalized by some speculations on laser pulse control of HET presented in Section VII.

\section{PROLOGUE}

Standard ET is described as the transition from a single donor state into a single acceptor state, with the donor state potential energy surface (PES) written as $E_{D}+U_{D}(Q)$ and that of the acceptor state as $E_{A}+U_{A}(Q)$. (Be aware of the notation used throughout the paper where the energy of the PES at the vibrational coordinate equilibrium position has been separated, here $E_{D}$ or $E_{A}$. $Q$ denotes the set of vibrational coordinates.) As it is well known, the electronic coupling strength $V_{D A}$ and the mutual position of the donor and acceptor PES (leading to a fixation of the driving force and the reorganization energy) are crucial for the concrete type of ET (adiabatic or nonadiabatic ET, as well as ET of the normal, activation 
FIG. 1: PES of the molecule semiconductor system $E_{a}+U_{a}$ versus a single vibrational coordinate $Q$ (the vertical position of the PES is determined by the electronic energy $E_{a}$, here without the inclusion of the vibrational zero-point energy). $a=g$ and $a=e$ correspond to the ground and excited state of the molecule, whereas $a=\mathbf{k}$ characterizes the semiconductor conduction-band states. The grey box indicates the continuum of band states starting at the lower band edge with energy $E_{\mathrm{con}}$. The position of the excited state PES $E_{e}+U_{e}$ drawn by a full line is typical for perylene on $\mathrm{TiO}_{2}$ where as that drawn by a dashed line resembles a near band-edge position.

less, or inverted region, cf., e.g., Refs. [4, 5]).

In contrast to this standard picture of ET the peculiarity of HET from a surface attached molecule into a semiconductor consists in the presence of a continuum of acceptor states formed by the conduction-band continuum. This is shown in Fig. 1, where we reduced the description to the presence of the electronic ground-state and a single excited state of the molecule with PES $E_{g}+U_{g}(Q)$ and $E_{e}+U_{e}(Q)$, respectively. The semiconductor has been described by a single conduction band. The electron in the excited state of the molecule may be transfered into the semiconductor since its empty conduction band is degenerate with the excited molecular level. Thus, the accepting levels of the ET are formed by the continuous band-structure $E_{\mathbf{k}}$ of the semiconductor, where $\mathbf{k}$ denotes the quasi-momentum (if a nano-cluster is considered $\mathbf{k}$ has to be replaced by other quantum numbers). At the 
same time the vibrational motion of the ionized molecule is determined by its cationic PES denoted in Fig. 1 as $U_{\text {ion }}$. Therefore the complete PES $E_{\mathbf{k}}+U_{\text {ion }}$ form a continuum, too, indicated by the shaded area in Fig. 1 (see also Section III A).

A HET scheme characterizing exclusively the electronic levels is given in Fig. 2. It presents the electronic configuration of the molecule-semiconductor system based on the introduction of a pseudo potential. Shown is the resulting overall potential following from the superposition of local potentials which define the local molecular or atomic electron levels which are also shown. The left well of the potential corresponds to the surface attached molecule, with the energetic position $E_{g}$ of the electronic ground-state and $E_{e}$ of the first excited state indicated. The wells in the right part of Fig. 2 are those formed by the various atoms of the semiconductor cluster. Here, the energetic positions $E_{m \mu}$ of the highest occupied and $E_{m \nu}$ of the lowest unoccupied atomic orbitals are drawn ( $m$ is the site-index and counts the various atoms, the level indices $\mu$ and $\nu$ have been not used in the figure). If the electronic eigenstates of the semiconductor are introduced one obtains the band structure with bulk and surface states. (Of course this differentiation becomes meaningless when considering a semiconductor nano-cluster.) The shown overall electronic configuration corresponds to an injected electron at the first atomic well leaving behind the cationic state of the molecule.

To characterize charge injection in more detail let us turn back to the PES scheme of Fig. 1. As it has been already mentioned, the mutual position of the donor (here the photo excited molecular state) and the acceptor PES fixes the type of ET (normal ET, activation less ET, or ET of the inverted region). In the present case of HET, however, all types may appear simultaneously. This is particularly the case if the injecting level is in a mid-band position (far away from the band edges of the semiconductor conduction band, full line in Fig. 1). Now, the donor PES has arbitrary crossing points with the multitude of acceptor PES, i.e. ET appears at every part of the donor PES.

If the ET is ultrafast as it is the case for perylene on $\mathrm{TiO}_{2}$ one has to consider $\mathrm{ET}$ in terms of vibrational wave packets. The fs-photo excitation results in a vibrational wave packet in the PES $U_{e}$ moving forth and back. Simultaneously, at every step of this motion a transfer to the acceptor PES is possible. Therefore, the decay of the overall donor population denoted here by $P_{e}$ will be smooth and structureless. But $P_{e}$ should show oscillations superimposed, if the injection level is in a near band edge position (dashed curve in Fig. 1). This results from the fact that in this case only a part of the donor PES crosses with the multitude of 


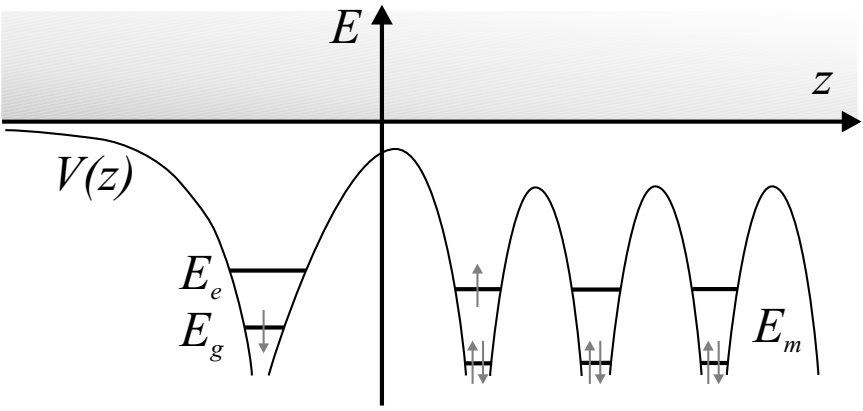

FIG. 2: Electronic levels of the molecule-semiconductor system according to a description by a one-dimensional pseudo potential $V(z)$. The left potential well belongs to the surface attached molecule and includes the ground-state with energy $E_{g}$ and the excited state with energy $E_{e}$. The following wells correspond to the atoms of the semiconductor at sites $m$ with atomic orbital energies $E_{m}$ (the quantum number which distinguishes the different levels has been suppressed). Shown is the electronic configuration after charge injection. The transferred electron occupies the empty atomic orbital of the first atom at the surface (notice that in the general case the level position depends on their actual population). The grey shaded area of the upper part $(E>0)$ indicates the region where unbound (vacuum state) electronic wave functions are defined.

acceptor PES.

Lets turn back to HET with the molecular injection level in a rather mid-band position. For this case Fig. 3 shows the excited molecule PES and some PES of the continuum of product states. For every PES the energetic position of the vibrational eigenstates are also shown (here levels corresponding to a single coordinate). The vibrational levels of the PES $E_{\mathbf{k}}+U_{\text {ion }}$ of the band continuum which have been drawn in the figure are degenerated with the levels of the molecular PES $E_{e}+U_{e}$. Thus, the scheme indicates that completely resonant transitions are possible from a particular excited molecular electron-vibrational level into different vibrational levels of the molecular cation with the electron in a band state.

These transitions are the only one which remain if the transfer coupling is weak and the Golden Rule of quantum mechanics suffices to describe the transition. Consequently, electronic band states of the semiconductor are populated around the injection energy $E_{e}$ shifted by multiples of the vibrational quanta. For stronger transfer coupling, however, also transitions into states are possible which are not completely degenerated with the excited molecular level. Nevertheless we may expect again structures around $E_{e}$ reflecting the 


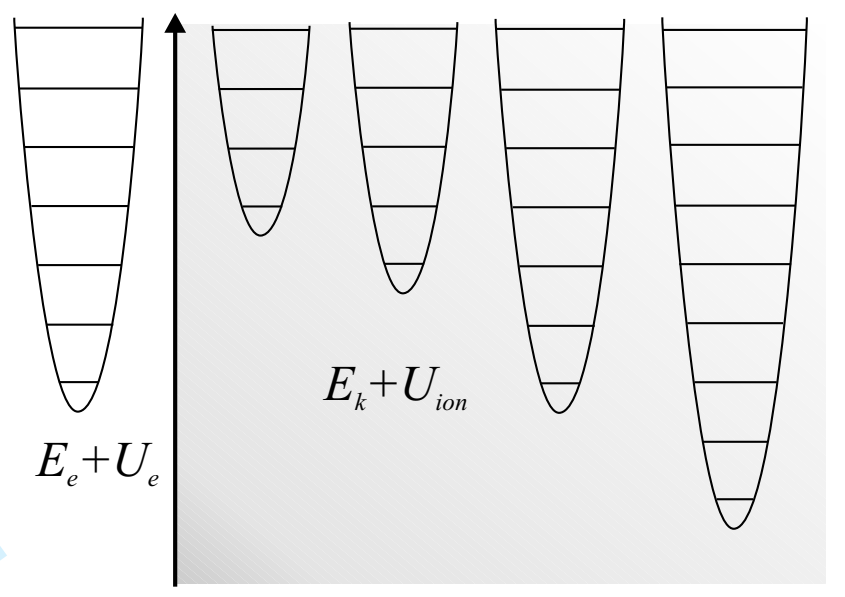

FIG. 3: PES $E_{e}+U_{e}$ of the excited molecular state (left) and some selected PES $E_{\mathbf{k}}+U_{\text {ion }}$ corresponding to the molecular cation and the injected electron at a particular band state (right part, the mutual horizontal shifts have been introduced for clearness). All PES are drawn together with the respective level position of vibrational eigenstates. The PES in the right part all act as acceptor levels and may be simultaneously addressed in the transfer process.

vibrational progression of the molecule but, now, with a broadening which also reflects the strength of transfer coupling (see Section IV A).

While the discussion done so far concentrated on the temporal evolution of the HET next we will consider the effect of HET on steady state properties like the linear absorbance, thus shifting the discussion to frequency domain considerations (cf. Section V). Neglecting vibrational contributions first we arrive at the scheme of a single excited molecular level (with energy $E_{e}$ ) coupled to the continuum of conduction band levels (with energy $E_{\mathbf{k}}$ ). Accordingly one expects a shift and a broadening of the molecular absorbance. Taking the vibrational degrees of freedom into account it not only depends on the coupling strength to the band continuum but also on the mutual position of the PES (the reorganization energy of the charge injection) if a vibrational progression seen at the isolated molecule survives its attachment to the semiconductor surface.

All the given qualitative discussions will be substantiated in the following section by respective quantitative consideration based on a uniform theory of photoinduced ultrafast HET. 


\section{THE MODEL}

The consideration of the molecule-semiconductor system is based on a diabatic-state like description distinguishing between molecular and semiconductor states (cf. Fig. 2). Of course, when carrying out ab initio calculations diabatic states are not directly obtained and a diabatization procedure becomes necessary. But their introduction is very useful for the consideration presented in the following. It is based on separate molecular states and those of the semiconductor. Both types of states couple one to another by a transfer coupling. The electronic states of the molecule are denoted as $\phi_{a}$ for the neutral one and as $\phi_{a}^{(+)}$for the states of the molecular cation. The quantum number $a$ counts the different levels with $a=g$ for the ground-state $a=e$ for the first excited state $\left(S_{1}\right.$-state) and, if necessary, $a=f$ for a higher excited state. This counting system is also used for the cationic states.

As the electronic states of the semiconductor we may introduce the band-states $\phi_{\alpha \mathbf{k}}$ where $\alpha$ is the band-index and $\mathbf{k}$ the quasi-wave vector of bulk or surface states. The filled valence band acting as reference state for all what follows is denoted as $|v\rangle$ (in the most simple view it can be considered as the antisymmetrized product state formed by the various single electron valence-band state functions $\phi_{v \mathbf{k}}$ ). When considering nano-crystals (clusters), however, it is more appropriate to work with localized states. Here, it is sufficient to use a single-electron description based on a tight-binding model with local atomic orbitals $u_{m \mu}^{(-)}$, with energies $E_{m \mu}$, and with the transfer coupling $T_{m \mu, n \nu}$. The notation accounts for the site $m$ of the atom and its orbital $\mu$ populated by the injected (excess) electron. The minus-sign at the atomic orbitals indicates that they refer to an excess electron injected into the prior neutral cluster.

For the whole characterization of the molecule-semiconductor system we have to introduce (antisymmetrized) electronic product states $\left|\phi_{a}\right\rangle|v\rangle$ and $\left|\phi_{a}^{(+)}\right\rangle\left|u_{m \mu}^{(-)}\right\rangle$. The first type describes the system before charge injection and the latter type corresponds to the states after charge injection. Here, $\left|u_{m \mu}^{(-)}\right\rangle$has to be understood as a many-electron state with the injected excess electron in the atomic orbital $u_{m \mu}^{(-)}$(the many-electron state could be given by the antisymmetrized product of $u_{m \mu}^{(-)}$and $\left.|v\rangle\right)$.

If we expand the total Hamiltonian $H_{\text {mol-sem }}$ of the molecule-semiconductor system with 
respect to these product states we obtain

$$
\begin{aligned}
& H_{\text {mol-sem }}=\sum_{a}\left(E_{v}+E_{a}+H_{a}\right)\left|\phi_{a}, v\right\rangle\left\langle v, \phi_{a}\right| \\
& +\sum_{a} \sum_{m \mu, n \nu}\left(\delta_{m \mu, n \nu}\left(E_{v}+E_{a}^{(+)}+H_{a}^{(+)}+E_{m \mu}^{(-)}\right)\right. \\
& \left.+T_{m \mu, n \nu}\right)\left|\phi_{a}^{(+)}, u_{m \mu}^{(-)}\right\rangle\left\langle u_{n \nu}^{(-)}, \phi_{a}^{(+)}\right| \\
& +\sum_{a} \sum_{b, n \nu}\left(V_{a, b n \nu}\left|\phi_{a}, v\right\rangle\left\langle u_{n \nu}^{(-)}, \phi_{a}^{(+)}\right|+\text {h.c. }\right)
\end{aligned}
$$

This expression needs some comments. First, notice that $E_{v}$ is the energy of the filled valence states which serves as a reference energy. Moreover, the $E_{a}+H_{a}$ characterize the neutral molecule with $E_{a}$ as the electronic energy at the equilibrium configuration of the respective nuclear coordinate PES, here, plus the zero-point energy. Correspondingly, $H_{a}$ denotes the respective vibrational Hamiltonian which spectrum starts at zero energy. The vibrational eigenstates are denoted as $\chi_{a M}$ ( $M$ comprises the vibrational quantum numbers). After charge injection the molecular cation is described by $E_{a}^{(+)}+H_{a}^{(+)}$defined in a identical way as for the neutral molecule. These molecular energies are combined with the energies $E_{m \mu}^{(-)}$ of the atomic orbital occupied by the injected electron (all related to the reference energy $\left.E_{v}\right)$.

ET of the injected electron within the nano-cluster is initiated by the transfer coupling $T_{m \mu, n \nu}$. The transfer coupling between the molecule and the semiconductor is accounted for by $V_{a, b n \nu}$ indicating transfer between the neutral molecular state $\phi_{a}$ and the atomic orbital $u_{n \nu}^{(-)}$leaving behind the cationic state $\phi_{b}^{(+)}$. Clearly, only a very selected set of atoms positioned around the binding site of the molecule is included. To avoid overloading of the model we neglected vibrational modulation of $V_{a, b n \nu}$. Of course, this assumption has to be proven when changing to a concrete molecule-semiconductor system. If photo emission of an electron from a semiconductor state into the vacuum is of interest the description has to be extended by the $\phi_{\kappa}^{(-)}$representing states which change into plane waves of a free electron for large distances with respect to the semiconductor cluster (grey shaded area in Fig. 2).

So far any Coulombic interaction of the injected electron with the positively charged molecule has been neglected. Often one argues that the diabatization necessary to arrive at the used local states already accounts for this coupling. We will adopt this position here, too. To include optical excitation $H_{\text {mol-sem }}$, Eq. (1) has to be complemented by the standard 
expression

$$
H_{\text {field }}=-\mathbf{E}(t) \hat{\mu} \text {. }
$$

The electric field--strength is denoted by $\mathbf{E}$, and $\hat{\mu}$ is the transition dipole operator which may account for an exclusive excitation of the molecule but also for charge transitions from the molecular ground-state into the semiconductor band states as well as photo ionization transitions.

\section{A. Reference Model}

The model introduced so far will be specified in the following to the description that we have already applied in our former studies [24-29]. Therefore, we introduce a notation by concentrating on the state of the single electron to be transferred. We also change to a common description of the semiconductor states. This results in an identification of the states before charge injection by $\varphi_{a}(a=g, e)$ and afterwards by $\varphi_{\mathbf{k}}$, i.e. the following assignment is taken: $\left|\phi_{a}, v\right\rangle \rightarrow\left|\varphi_{a}\right\rangle$ and $\left|\phi_{a}^{(+)}, \phi_{\nu \mathbf{k}}\right\rangle \rightarrow\left|\varphi_{\mathbf{k}}\right\rangle$. The respective electronic energies are $\hbar \varepsilon_{a}$, here with $a$ also including the quasi-wave vector $\mathbf{k}$ as an electronic quantum number (notice that $E_{v}$ has been set equal to zero). It is not required to distinguish whether the $\varphi_{\mathbf{k}}$ are bulk or surface states since we use for concrete computations the density of states (DOS)

$$
\mathcal{N}(\Omega)=\sum_{\mathbf{k}} \delta\left(\Omega-\omega_{\mathbf{k}}\right)
$$

Here and in the following $\hbar \Omega$ labels the semiconductor band energy. When choosing a particular form of $\mathcal{N}(\Omega)$ it should cover all semiconductor band states in the vicinity of the molecular injection level. Probably, the use of an averaged DOS

$$
\overline{\mathcal{N}}=\frac{N_{\Omega}}{\Delta \Omega}
$$

with the level number $N_{\Omega}$ in the energy interval $\Delta \Omega$ would be sufficient (wide-band approximation).

According to what has been discussed beforehand the Hamiltonian, Eq. (1) reduces to

$$
\begin{aligned}
H_{\text {mol-sem }}= & \sum_{a=g, e, \mathbf{k}}\left(\hbar \varepsilon_{a}+H_{a}\right)\left|\varphi_{a}\right\rangle\left\langle\varphi_{a}\right| \\
& +\sum_{\mathbf{k}}\left(V_{\mathbf{k} e}\left|\varphi_{\mathbf{k}}\right\rangle\left\langle\varphi_{e}\right|+\text { h.c. }\right) .
\end{aligned}
$$




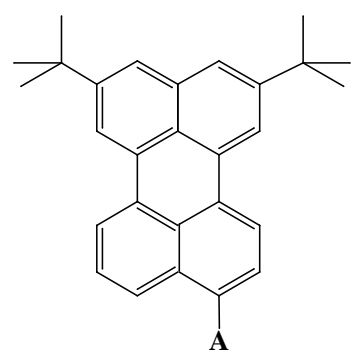<smiles>CC(=O)O</smiles>

(1)<smiles>C/C=C/C(=O)O</smiles>

(2)<smiles>CCCC(=O)O</smiles>

(3)

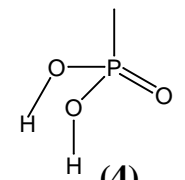

(4)

FIG. 4: Core chromophore DTB-Pe-A (di-tertiary-butyl perylene, upper panel) with bridgeanchor groups (lower panel) in position A as studied in Ref. [28]. 1: - $\mathrm{COOH}$ (carboxylic acid), 2:$(\mathrm{CH})_{2}-\mathrm{COOH}$ (acrylic acid), $3-\left(\mathrm{CH}_{2}\right)_{2}-\mathrm{COOH}$ (proprionic acid), and 4: $-\mathrm{P}(\mathrm{O})(\mathrm{OH})_{2}$ (phosphonic acid).

For the further discussion it is useful to write the band energies $\hbar \varepsilon_{\mathbf{k}}$ as $\hbar \omega_{\text {con }}+\hbar \omega_{\mathbf{k}}$, with the lower band-edge $\hbar \omega_{\text {con }}$ and with $\hbar \omega_{\mathbf{k}}$ running over the conduction band of width $\hbar \Delta \omega_{\text {con }}$. Again, all levels are understood as given by the minima of the related PES plus the zero-point vibrational energy. $H_{g}$ and $H_{e}$ denote the vibrational Hamiltonian (with a spectrum starting at zero energy) for the electronic ground-state and the first excited state of the molecule, respectively. $H_{\text {ion }}$ is the one of the ionized molecule if charge injection into the conduction band took place (earlier denoted as $H_{g}^{(+)}$). The related eigenvalues and vibrational wave functions have been already introduced. Charge injection from $\varphi_{e}$ into the manifold of states $\varphi_{\mathbf{k}}$ is realized by the transfer coupling $V_{\mathbf{k} e}$ which $\mathbf{k}$-dependence will be later replaced by a frequency-dependence leading to $V_{e}(\Omega)$ (within the wide-band approximation it can be replaced by the frequency independent, averaged quantity $\left.\bar{V}_{e}\right)$.

If we assume that optical excitation exclusively takes place in the molecule between the ground and the excited state the dipole operator introduced in Eq. (2) reads

$$
\hat{\mu}=\mathbf{d}_{e g}\left|\varphi_{e}\right\rangle\left\langle\varphi_{g}\right|+\text { h.c. }
$$

with the transition-dipole matrix element denoted as $\mathbf{d}_{e g}$. If a direct excitation of conduction-band states is considered the additional contribution

$$
\hat{\mu}_{\mathrm{CT}}=\sum_{\mathbf{k}} \mathbf{d}_{\mathbf{k} g}\left|\varphi_{\mathbf{k}}\right\rangle\left\langle\varphi_{g}\right|+\text { h.c. }
$$

has to be included $\left(\mathbf{d}_{\mathbf{k} g}\right.$ might be replaced by $\left.\mathbf{d}_{g}(\Omega)\right)$. 
TABLE II: Parameters of the DTB-Pe- $\left(\mathrm{CH}_{2}\right)_{2}-\mathrm{COOH}$ system at the $\mathrm{TiO}_{2}$ surface.

\begin{tabular}{cc}
\hline \hline$\varepsilon_{e}$ & $2.79 \mathrm{eV}$ \\
$\hbar \omega_{\mathrm{vib}}$ & $0.17 \mathrm{eV}$ \\
$\lambda_{e g},\left(Q_{e}-Q_{g}\right)$ & $0.187 \mathrm{eV},(2.1)$ \\
$d_{e g}$ & $3 \mathrm{D}$ \\
$\hbar \gamma$ & 0.058 \\
$\hbar \varepsilon_{\text {con }}$ & $1.79 \mathrm{eV}$ \\
$\hbar \Delta \omega_{\text {con }}$ & $6.0 \mathrm{eV}$ \\
$\hbar \bar{\Gamma}$ & 0.0213 \\
$\bar{V}_{e}(\overline{\mathcal{N}} / \hbar)$ & $0.058 \mathrm{eV},(2 / \mathrm{eV})$ \\
$\lambda_{\text {ion } e},\left(Q_{\text {ion }}-Q_{e}\right)$ & $0.014 \mathrm{eV},(-0.6)$ \\
\hline \hline
\end{tabular}

\section{B. The Perylene $\mathrm{TiO}_{2}$ System}

We shortly comment here on the specification of the injection model discussed in the preceding section to the perylene $\mathrm{TiO}_{2}$ system. It is based on our recent studies which considered the steady state absorption spectra of perylene attached to $\mathrm{TiO}_{2}$ nano-crystals 
via different bridge-anchor groups (cf. Ref. [28]). Changing the bridge-anchor groups one may change the transfer coupling and thus the injection time. In this way one probably changes a single parameter of the injection model what would be of large interest for a systematic study.

The different bridge-anchor groups which have been investigated so far in [28] are shown in Fig. 4 together with the perylene molecule. The DTB-Pe-COOH-TiO 2 and DTB-Pe$(\mathrm{CH})_{2}-\mathrm{COOH}-\mathrm{TiO}_{2}$ system are characterized by a stronger transfer coupling in contrast to the DTB-Pe-P $(\mathrm{O})\left(\mathrm{OH}_{2}\right)_{2}-\mathrm{TiO}_{2}$ and DTB-Pe- $\left(\mathrm{CH}_{2}\right)_{2}-\mathrm{COOH}-\mathrm{TiO}_{2}$ system. Respective parameters deduced from a fit of the absorption spectra are shown for the first system (with a carboxylic acid bridge -anchor group) in Table I and the last mentioned system (with the proprionic acid bridge-anchor group) in table II (the respective theoretical background is presented in Section V). Besides the electronic parameters (excited molecular level $\hbar \varepsilon_{e}$, lower conduction-band edge $\hbar \varepsilon_{\text {con }}$, band width $\hbar \omega_{\text {con }}$, averaged DOS $\overline{\mathcal{N}}$, Eq. (4), and transfer coupling $\bar{V}_{e}$ ) the tables contain the single vibrational frequency $\omega_{\text {vib }}$ and two reorganization energies $\lambda$ (for explanation see below) as well as the energy broadening $\hbar \bar{\Gamma}$ introduced in Section IV B and the the overall dephasing rate (see section V). For the transition-dipole matrix element $d_{e g}$ there does not exist a uniform value. We take 3 Debye (cf. [28]).

In general, several vibrational modes will contribute to the absorption spectrum of any aromatic chromophore and this holds true also in the case of perylene [36, 37]. The room temperature spectra, however, could be simulated rather well by a single-mode description. The spectra display the dominance of a single vibrational mode (also if the molecule is in a solvent) having a quantum energy $\hbar \omega_{\text {vib }}$ of about $0.17 \mathrm{eV}\left(1370 \mathrm{~cm}^{-1}\right)$ and corresponding to an in-plane $\mathrm{C}-\mathrm{C}$ stretching vibration. (Including more vibrational modes which couple to the electronic transition is possible and may improve the fit of the measured data. But at the same time this would require the introduction of many more parameters into the fit what makes the whole procedure rather ambiguous.)

The single-mode model of Ref. [28] has also been taken as a justification of the singlemode description already used in Refs. [24-27]. Therefore, the involved PES are denoted as $(a=g, e$, ion $)$ :

$$
U_{a}(Q)=\hbar \omega_{\mathrm{vib}}\left(\frac{1}{4}\left(Q-Q_{a}\right)^{2}-\frac{1}{2}\right)
$$

with the vibrational frequency $\omega_{\text {vib }}$ common to all considered electronic states. The notation removes the zero-point energy from the PES and is based on the use of a dimensionless 


\section{CHARGE INJECTION DYNAMICS}

Since the photoinduced dynamics are considered on a 100 fs time--window one can neglect any relaxational effect. Therefore, it is sufficient to propagate the time-dependent Schrödinger equation related to the Hamiltonian introduced in Eq. (5) together with the field-part, Eq. (2):

$$
i \hbar \frac{\partial}{\partial t}|\Psi(t)\rangle=\left(H_{\text {mol-sem }}+H_{\text {field }}(t)\right)|\Psi(t)\rangle .
$$

Its solution is based on an expansion with respect to the diabatic electron-vibrational states $\chi_{a M} \varphi_{a}(a=g, e, \mathbf{k})$ :

$$
|\Psi(t)\rangle=\sum_{a M} C_{a M}(t)\left|\chi_{a M}\right\rangle\left|\varphi_{a}\right\rangle .
$$

The given state expansion is fairly standard except for the presence of the band continuum leading to a continuous set $C_{\mathbf{k} M}(t)$ of expansion coefficients. We will tackle this problem as described in [24-29]. Therefore, the $\mathbf{k}$-dependence of the $C_{\mathbf{k} M}(t)$ is replaced by a frequency dependence leading to the quantities $C_{M}(\Omega ; t)$. They will be expanded by the functions $u_{r}(\Omega)$ forming an orthogonal set. The latter is complete with respect to the energy range of the conduction band, here characterized by the frequency interval $\left[0, \Delta \omega_{\text {con }}\right]$ (from the lower to the upper conduction band-edge). This allows us to write

$$
C_{M}(\Omega ; t)=\sum_{r} u_{r}(\Omega) C_{M}^{(r)}(t)
$$

An appropriate truncation of the infinite sum leads to a finite set of expansion coefficients $C_{M}^{(r)}(t)$. 
Accordingly, the coupled equations of motion for the expansion coefficients $C_{a M}$ introduced in Eq. (11) take the following form:

$$
\begin{aligned}
\frac{\partial}{\partial t} C_{g M}(t)= & -i\left(E_{g} / \hbar+\omega_{M}\right) C_{g M}(t) \\
& +\frac{i}{\hbar} \mathbf{E}(t) \mathbf{d}_{g e} \sum_{N}\left\langle\chi_{g M} \mid \chi_{e N}\right\rangle C_{e N}(t), \\
\frac{\partial}{\partial t} C_{e M}(t)= & -i\left(E_{e} / \hbar+\omega_{M}\right) C_{e M}(t) \\
+ & \frac{i}{\hbar} \mathbf{E}(t) \mathbf{d}_{e g} \sum_{N}\left\langle\chi_{e M} \mid \chi_{g N}\right\rangle C_{g N}(t) \\
- & \frac{i}{\hbar} \sum_{p} \sum_{N}\left\langle\chi_{e M} \mid \chi_{i o n}\right\rangle<\mathcal{N} V_{e} u_{p}>_{\Omega} \\
& \times C_{N}^{(p)}(t),
\end{aligned}
$$

and

$$
\begin{aligned}
\frac{\partial}{\partial t} C_{M}^{(r)}(t)= & -i\left(E_{\mathrm{c}} / \hbar+\omega_{M}\right) C_{M}^{(r)}(t) \\
& -i \sum_{p}<\Omega u_{r} u_{p}>_{\Omega} C_{M}^{(p)}(t) \\
& -\frac{i}{\hbar} \sum_{N}<u_{r} V_{e}>_{\Omega}\left\langle\chi_{i o n M} \mid \chi_{e N}\right\rangle \\
& \times C_{e N}(t) .
\end{aligned}
$$

The bracket $<\ldots>_{\Omega}$ denotes frequency integration according to $<u_{r} u_{p}>_{\Omega} \equiv \int_{0}^{\Delta \omega_{\text {con }}} d \Omega$ $u_{r}(\Omega) u_{p}(\Omega)=\delta_{r, p}$. This relation also indicates orthonormalization of the functions $u_{r}(\Omega)$. The expressions $<\mathcal{N} V_{e} u_{p}>_{\Omega}$ and $<u_{r} V_{e}>_{\Omega}$ account for the frequency dependence of the DOS and the transfer coupling.

For the concrete calculations we used a particular realization of the orthonormal set $u_{r}$. It is given by the Legendre polynomials $P_{r}$ according to the identification $u_{r}(\Omega)=$ $\sqrt{(2 r+1) / \Delta \omega_{\text {con }}} P_{r}(x(\Omega))$ with $x(\Omega)=2 \Omega / \Delta \omega_{\text {con }}-1$. The necessary number of the polynomials did not exceed 200 whereas the upper vibrational number was $M=22$. Such a large number of polynomials is necessary to achieve convergence with respect to the broad frequency distribution of the expansion coefficients Eq. (12) (see also Fig. 6).

Once the Eqs. (13) - (15) have been solved different observables can be computed. The 
populations of the molecular states follow as $(a=g, e)$

$$
P_{a}=\sum_{M}\left|C_{a M}(t)\right|^{2}
$$

That of the ionized state my be obtained from $P_{\text {ion }}(t)=1-P_{g}-P_{e}$ or directly as

$$
P_{\mathrm{ion}}(t)=\sum_{\mathbf{k}, M}\left|C_{\mathbf{k} M}(t)\right|^{2} \equiv \int d \Omega \mathcal{N}(\Omega) P_{\mathrm{el}}(\Omega ; t) .
$$

Here we introduced the distribution versus the band states

$$
\begin{aligned}
& P_{\mathrm{el}}(\Omega ; t)=\sum_{M}\left|C_{M}(\Omega ; t)\right|^{2} \\
& =\sum_{r, p} u_{r}(\Omega) u_{p}(\Omega) \sum_{M} C_{M}^{(r) *}(t) C_{M}^{(p)}(t) .
\end{aligned}
$$

Notice that $P_{\mathrm{el}}(\Omega ; t)$ may become larger than 1 for particular values of $\Omega$. The relation $P_{\text {ion }} \leq 1$, however, is guaranteed by the concrete form of the $\operatorname{DOS} \mathcal{N}(\Omega)$.

\section{A. Femtosecond Photoinduced Electron Transfer}

In order to characterize the ultrafast charge injection process we present the solution of the time-dependent Schrödinger equations (13) - (15) starting at the vibrational ground-state $\chi_{g 0}$ of the electronic ground-state and including a laser-field of 10 fs duration (FWHM). Moreover, the wide band-approximation has been used, thus, replacing $<\mathcal{N} V_{e} u_{p}>_{\Omega}$ and $<u_{r} V_{e}>_{\Omega}$ in Eqs. (14) and (15) by $\delta_{p, 0} \sqrt{\Delta \omega_{\text {con }}} \overline{\mathcal{N}} \bar{V}_{e}$ and $\delta_{r, 0} \sqrt{\Delta \omega_{\text {con }}} \bar{V}_{e}$, respectively.

Resulting charge injection dynamics related to two of the four bridge-anchor groups described in Section III B (cf. Table I and II) are displayed in Fig. 5. In the strongcoupling case the excited-state population $P_{e}$ follows the laser pulse envelope accompanied by a direct charge transfer into the conduction band continuum. The respective overall band population is identical with the population of the ionized molecular state $P_{\text {ion }}$. Here, one may consider the laser pulse excitation as a direct population of the semiconductor states. In the other case with a weaker coupling the excited-state population starts to decay into the band continuum when the laser pulse excitation is over, indicating the separation of excited state preparation and charge injection.

To characterize the time evolution of the injected electron in more detail we consider the probability distribution $P_{\mathrm{el}}(\Omega ; t)$ of the electron in the band continuum, Eq. (18). Results 

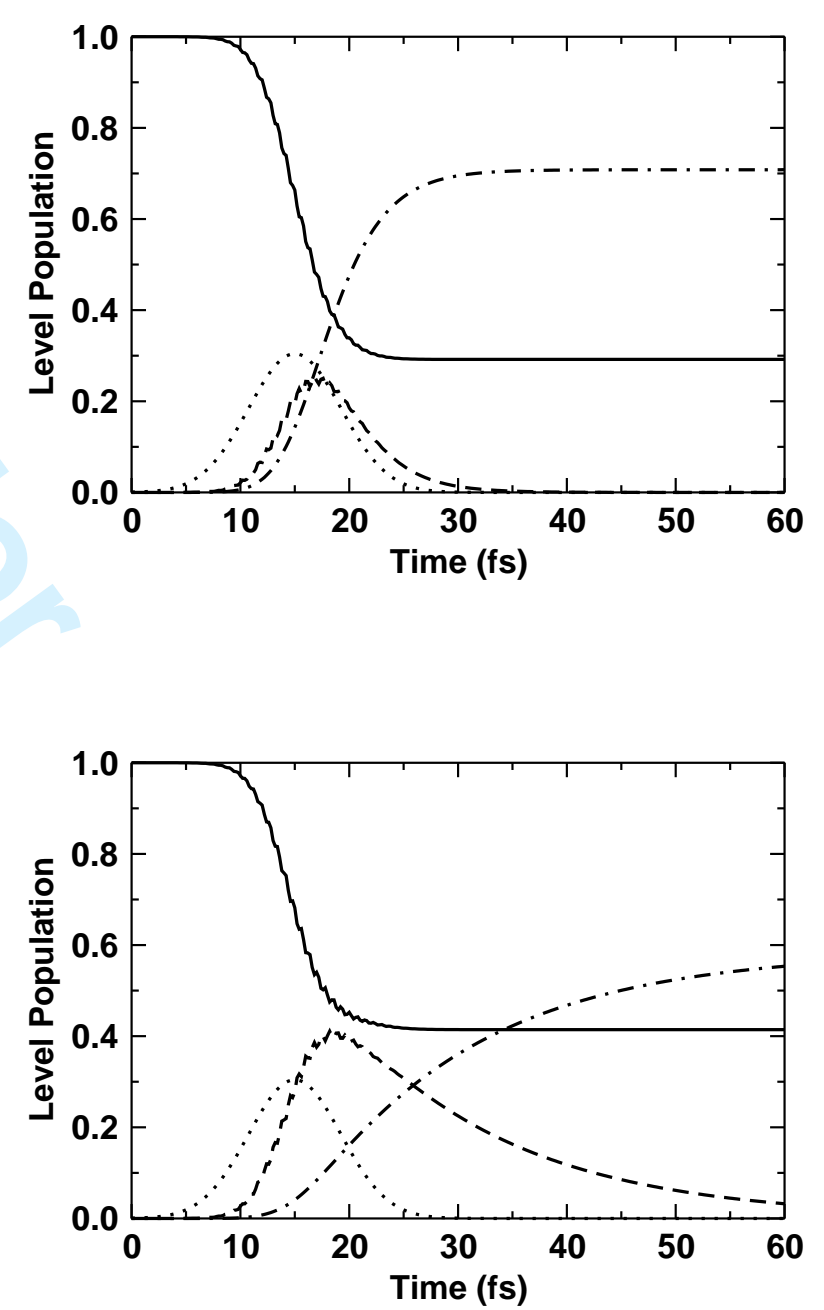

FIG. 5: Electronic level population after a $10 \mathrm{fs}$ (FWHM) laser pulse excitation. Upper panel: the DTB-Pe-COOH system (for parameters see Table I), lower panel: the DTB-Pe- $\left(\mathrm{CH}_{2}\right)_{2}-\mathrm{COOH}$ system (for parameters see Table II). Solid line: ground-state population $P_{g}$ of the molecule, dashed line: excited-state population $P_{e}$ of the molecule, dashed-dotted line: population $P_{i o n}$ of the ionized molecular state (what equals the total conduction band population), dotted line: shape of the laser pulse envelope (arbitrary units).

are shown in Fig. 6 for the two bridge-anchor groups presented in Fig. 5. It has already been indicated in Ref. [24] that $P_{\mathrm{el}}(\Omega ; t)$ displays the vibrational progression of the involved coordinate. Fig. 6 demonstrates that after a certain time interval (reflecting energy-time uncertainty) the broad distribution decays into different peaks. They correspond to tran- 
sitions from the excited molecular state with energy $\hbar \varepsilon_{e}+\hbar \omega_{e M}$ into the conduction-band continuum with energy $\hbar \omega_{\text {con }}+\hbar \Omega+\hbar \omega_{N}$. The possible energy values $\hbar \Omega$ in the band follow as $\hbar \varepsilon_{e}-\hbar \omega_{\text {con }}+\hbar\left(\omega_{e M}-\omega_{N}\right)$ reflecting an inelastic charge injection accompanied by the creation or destruction of quanta of the vibrational coordinate (cf. also Fig. 3). If the vibrational ground-state of the excited molecular state would be populated only, $P_{\mathrm{el}}(\Omega ; t)$ should extend to an energy range below $\hbar \varepsilon_{e}-\hbar \omega_{\text {con }}$. The simultaneous population of excited vibrational states may cause also structures in $P_{\text {el }}(\Omega ; t)$ above $\hbar \varepsilon_{e}$. This would be the case after an ultrashort optical excitation. In Fig. 6 it is less obvious since the vibrational energy is larger than $0.1 \mathrm{eV}$ (cf. Tables I and II). Notice the similarity with the linear absorbance discussed in Section $\mathrm{V}$ where also a strong transfer coupling leads to a strong broadening of the vibrational progression (compared with the case of the dissolved molecule) and a weak coupling to a less pronounced broadening. In any case, the whole energetic extension of $P_{\text {el }}(\Omega ; t)$ reflects the distribution of Frank-Condon overlap integrals $\left\langle\chi_{\text {ion } N} \mid \chi_{e 0}\right\rangle$. We also emphasize that the energetic dispersion of $P_{\text {ion }}$, Eq. (17), by introducing $P_{\mathrm{el}}(\Omega ; t)$ resolves vibrational state contributions. Those become observable although the HET proceeds on a time scale of some femtoseconds. Analyzing exclusively $P_{\text {ion }}$ such detailed information would be not available.

\section{B. Decay into the Band Continuum}

The numerical results of the foregoing section are complemented by analytical calculations referring to the decay of the population $P_{e M}(t)$ of an excited molecular electron-vibrational state upon charge injection (starting at $t=0$ ). These calculations will be based on the Green's operator

$$
\hat{G}(t)=-i \Theta(t) e^{-i H_{\mathrm{mol}-\mathrm{sem}} t / \hbar},
$$

defined by the total time-evolution operator of the molecule semiconductor system. $P_{e M}(t)$ is the survival probability of the initially prepared state, and one immediately obtains

$$
\begin{aligned}
& P_{e M}(t)=\left|\left\langle\varphi_{e} \chi_{e M}|\hat{G}(t)| \chi_{e M} \varphi_{e}\right\rangle\right|^{2} \\
& \equiv\left|\int \frac{d \omega}{2 \pi} e^{-i \omega t} G_{e M, e M}(\omega)\right|^{2} .
\end{aligned}
$$

Detailed considerations of the Green's operator, of its Fourier-transformation, and of its electron-vibrational matrix elements can be found in Appendix A. As a main ingredient of 


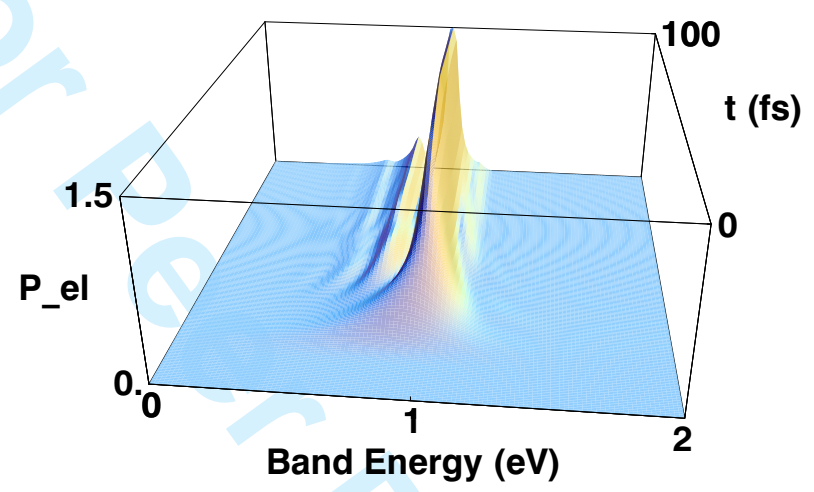

FIG. 6: Probability distribution of the injected electron $P_{\mathrm{el}}(\Omega ; t)$, Eq. (18) versus the energy $\hbar \Omega$ within the conduction band and versus time (the origin of the energy axis is given by $\hbar \omega_{\text {con }}$, the excitation conditions and parameters are identical with those of Fig. 5). Upper panel: the DTB$\mathrm{Pe}-\mathrm{COOH}$ system (for parameters see Table I), lower panel: the DTB-Pe- $\left(\mathrm{CH}_{2}\right)_{2}-\mathrm{COOH}$ system (for parameters see Table II).

these computations the self-energy due to the coupling of the excited molecular level to the band continuum appears:

$$
\Sigma(\omega)=\frac{1}{\hbar^{2}} \sum_{\mathbf{k}} \frac{\left|V_{\mathbf{k} e}\right|^{2}}{\omega-\varepsilon_{\mathbf{k}}+i \epsilon} .
$$

The imaginary part of $\Sigma(\omega)$ can be found in Eq. (A15) and will be denoted here in using the DOS, Eq. (3), and by changing from $V_{\mathbf{k} e}$ to $V_{e}(\Omega)$ :

$$
-\operatorname{Im} \Sigma(\omega)=\Gamma(\omega)=\frac{\pi}{\hbar^{2}} \mathcal{N}(\omega)\left|V_{e}(\omega)\right|^{2}
$$

In the general case an analytical expression for $P_{e M}(t)$, Eq. (20) is hardly obtainable. Applying, however, the wide-band approximation (where the frequency-dependence of the 
TABLE III: Comparison of charge injection times for all perylene bridge-anchor group $\mathrm{TiO}_{2}$ systems shown in Fig. 4: $\tau_{\text {inj }}^{(\text {exp) }}$ follows from a rate equation fit of measured transient absorption data (cf. Ref. [38]) and $1 / k_{\mathrm{HET}}$ is the inverse of the HET rate, Eq. (24).

\begin{tabular}{ccc}
\hline \hline & $\tau_{\text {inj }}^{(\exp )}(\mathrm{fs})$ & $1 / k_{\mathrm{HET}}(\mathrm{fs})$ \\
\hline DTB-Pe-COOH-TiO & 5 \\
DTB-Pe- $(\mathrm{CH})_{2}-\mathrm{COOH}-\mathrm{TiO}_{2}$ & 13 & 6 \\
DTB-Pe- $(\mathrm{CH})_{2}-\mathrm{COOH}-\mathrm{TiO}_{2}$ & 10 & 16 \\
DTB-Pe-P $(\mathrm{O})\left(\mathrm{OH}_{2}\right)_{2}-\mathrm{TiO}_{2}$ & 57 & 9 \\
\hline \hline
\end{tabular}

self-energy is neglected) the standard expression

$$
P_{e M}(t)=e^{-k_{\mathrm{HET}} t}
$$

follows with the rate of HET obtained as

$$
k_{\mathrm{HET}}=-2 \operatorname{Im} \bar{\Sigma} \equiv 2 \bar{\Gamma}=\frac{2 \pi}{\hbar^{2}} \overline{\mathcal{N}}\left|\bar{V}_{e}\right|^{2}
$$

$\overline{\mathcal{N}}$ denotes the averaged value of the DOS, Eq. 4 and $\bar{V}_{e}$ is the averaged transfer coupling. Respective values have been already given in Tables I and II. (Note that this wide-band approximation suppresses any vibrational contributions.) Since the transfer coupling connects the excited molecular state to some atoms of $\mathrm{TiO}_{2}$ around the binding site of perylene only, the used small DOS seems to be reasonable. A concrete value for $\overline{\mathcal{N}}$ can be deduced from the calculations of Ref. [35], since they have been restricted to rather small $\mathrm{TiO}_{2}$ cluster, i.e. to the localized states around the binding site.

Tab. III relates measured injection time constants $\tau_{\text {inj }}^{(\text {exp })}$ derived from data of the cation transient absorption to those obtained from the simulation of steady state absorption spectra (see next section). The latter are given as the inverse of the HET rates $k_{\mathrm{HET}}$, Eq. (24). The time constants obtained from the calculations reproduce the qualitative trend of the measured injection time constants. However, the $k_{\mathrm{HET}}$ are always too small what might be explained by the neglect of structural and energetic disorder (for a more detailed discussion see Section V C). 


\section{THE LINEAR ABSORPTION COEFFICIENT}

The consideration of linear absorption spectra is of particular importance since their detailed analysis offers a unique way to specify all parameters of our model (cf. [28]). The computation of linear absorption spectra of molecular systems represents a standard task (cf. e.g. [5]) and is based on the following expression:

$$
\alpha(\omega)=\frac{4 \pi \omega n_{\mathrm{mol}}}{\hbar c} \operatorname{Re} \int_{0}^{\infty} d t e^{i \omega t} C_{\mathrm{d}-\mathrm{d}}(t) .
$$

Here, $n_{\text {mol }}$ denotes the volume density of the absorbing molecules. The given formula relates the frequency dependent absorbance to the half-sided Fourier-transformed dipole-dipole correlation function given by

$$
C_{\mathrm{d}-\mathrm{d}}(t)=<\operatorname{tr}\left\{\hat{W}_{\text {eq }}[\hat{\mu}(t), \hat{\mu}]_{-}\right\}>_{\text {disorder }}
$$

The trace covers the summation with respect to all molecule-semiconductor system states. Averaging with respect to structural and energetic disorder was also introduced, symbolized by $\langle\ldots\rangle_{\text {disorder }}$. The thermal equilibrium state of the system before photo absorption is characterized by the statistical operator $\hat{W}_{\text {eq }}=\hat{R}_{g}\left|\varphi_{g}\right\rangle\left\langle\varphi_{g}\right|$, describing vibrational equilibrium (with density operator $\hat{R}_{g}$ ) in the electronic ground-state. The time-dependence of the dipole operator $\hat{\mu}(t)$ has been induced by the Hamiltonian $H_{\text {mol-sem }}$, Eq. (5). In the following we only account for random orientation of the molecules (leading to the well-known prefactor $1 / 3$, a somewhat more involved description is given below). Since non-Condon effects are of less importance for our further treatment we may write (cf. Eq. (6))

$$
\begin{aligned}
& C_{\mathrm{d}-\mathrm{d}}(t)=\frac{1}{3}\left|\mathbf{d}_{e g}\right|^{2} \\
& \operatorname{tr}_{\mathrm{vib}}\left\{\left\langle\varphi_{e}\left|e^{-i H_{\mathrm{mol}-\mathrm{sem}} t / \hbar} \hat{R}_{g}\right| \varphi_{e}\right\rangle\left\langle\varphi_{g}\left|e^{i H_{\mathrm{mol}-\mathrm{sem}} t / \hbar}\right| \varphi_{g}\right\rangle\right\} .
\end{aligned}
$$

The trace $\operatorname{tr}_{\mathrm{vib}}\{.$.$\} has to be taken with respect to the vibrational states, and anti-resonance$ contributions (resonances at negative frequencies) have been neglected.

Introducing the Greens operator, Eq. (19) and notice $\hat{G}_{e e}(t)=\left\langle\varphi_{e}|\hat{G}(t)| \varphi_{e}\right\rangle$ we get

$$
-i \Theta(t) C_{\mathrm{d}-\mathrm{d}}(t)=\frac{1}{3}\left|\mathbf{d}_{e g}\right|^{2} e^{i \varepsilon_{g} t} \operatorname{tr}_{\mathrm{vib}}\left\{\hat{G}_{e e}(t) \hat{R}_{g} e^{i H_{g} t / \hbar}\right\}
$$


This expression can be used as the starting point of time-domain or frequency-domain computations. The latter will be carried out in Section V C. Here, we present a time-domain description of the linear absorbance. Therefore, we concentrate on a situation where only the vibrational ground-state $\chi_{g 0}$ of the electronic ground-state is populated (low temperatures or exclusively high-frequency vibrational modes or both). Then, the trace with respect to the vibrational states in Eq. (28) can be written as

$$
\begin{aligned}
& \operatorname{tr}_{\mathrm{vib}}\left\{\hat{G}_{e e}(t) \hat{R}_{g} e^{i H_{g} t / \hbar}\right\}=\left\langle\chi_{g 0}\left|\hat{G}_{e e}(t)\right| \chi_{g 0}\right\rangle \\
& \equiv-i \Theta(t)\left\langle\chi_{g 0} \varphi_{e}\left|e^{-i H_{\mathrm{mol}-\mathrm{sem}} t / \hbar}\right| \varphi_{e} \chi_{g 0}\right\rangle
\end{aligned}
$$

indicating that the state vector $\left|\varphi_{e} \chi_{g 0}\right\rangle$ has to be propagated under the action of the complete Hamiltonian $H_{\text {mol-sem }}$, Eq. (5) (the absence of any ground-state excited-state coupling in $H_{\text {mol-sem }}$, however, eliminates any ground-state contribution). We introduce the expansion Eq. (11) with respect to the electron-vibrational states $\left|\chi_{a M}\right\rangle\left|\varphi_{a}\right\rangle$ (with $a$ restricted here to $e$ and $\mathbf{k}$ ) and carry out the time propagation by solving the Eqs. (14) and (15), The initial condition reads

$$
C_{a M}(0)=\delta_{a, e}\left\langle\chi_{e M} \mid \chi_{g 0}\right\rangle
$$

According to this treatment one obtains

$$
\left\langle\chi_{g 0}\left|\hat{G}_{e e}(t)\right| \chi_{g 0}\right\rangle=-i \Theta(t) \sum_{M}\left\langle\chi_{g 0} \mid \chi_{e M}\right\rangle C_{e M}(t) .
$$

Once this matrix element has been calculated we can carry out the partial Fouriertransformation in Eq. (25) for the absorption coefficient and get $\alpha(\omega)$ versus $\omega$. As it is well known this procedure avoids any calculation of system eigenstates and eigenfunctions. In particular, a full account for the frequency dependence of the DOS and the transfer integral is equivalent to a complete consideration of the self-energy, Eq. (21) (which, of course, is not calculated explicitly here).

\section{A. Perylene on $\mathrm{TiO}_{2}$}

The method of the foregoing section has been used to compute the absorbance related to perylene attached via different bridge-anchor groups to the surface of $\mathrm{TiO}_{2}$ nanocrystals. Respective experimental spectra are shown in Fig. 7 for the DTB-Pe-COOH- $\mathrm{TiO}_{2}$ and the DTB-Pe- $(\mathrm{CH})_{2}-\mathrm{COOH}-\mathrm{TiO}_{2}$ system as well as for the molecule in a solvent with the 


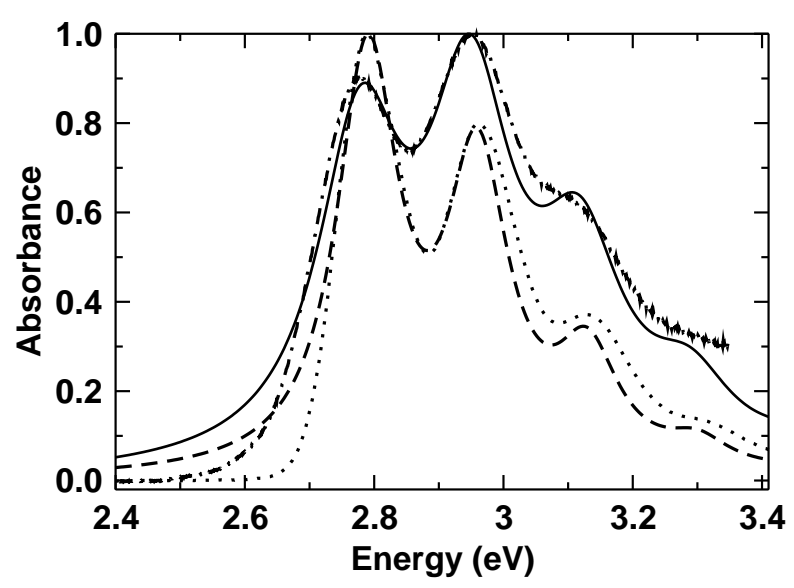

FIG. 7: Rescaled linear absorption spectrum of the DTB-Pe-COOH system (upper panel) and the DTB-Pe- $\left(\mathrm{CH}_{2}\right)_{2}-\mathrm{COOH}$ system (lower panel). Dotted lines: experimental data for the system in the solvent, dashed-dotted lines: experimental data for the system adsorbed at a $\mathrm{TiO}_{2}$ surface, dashed lines: calculated data for the system in the solvent, full lines: calculated data for the system adsorbed at a $\mathrm{TiO}_{2}$ surface (for the used parameters see Tables I and II).

respective bridge-anchor groups. The measured spectra for the molecules in a solvent show a vibrational progression which has been related to a perylene in-plane $\mathrm{C}-\mathrm{C}$ stretching vibration with quantum energy of $1370 \mathrm{~cm}^{-1}$ [28]. The $0-0$-transition as well as the $0-$ 1-, 0-2-, and 0-3-transition are clearly resolved. The solvent spectra have been used to fix some internal perylene parameters (energetic position of the excited state, vibrational 
energy, reorganization energy accompanying the excitation and overall dephasing rate, see Tables I and II).

Lets turn to the spectra of the molecules attached to the $\mathrm{TiO}_{2}$ surface. For the DTB-Pe$\mathrm{COOH}-\mathrm{TiO}_{2}$ system the vibrational progression found in the solvent is lost in the adsorbed state and an almost structureless absorption band appears instead. In contrast, the systems of DTB-Pe- $\left(\mathrm{CH}_{2}\right)_{2}-\mathrm{COOH}-\mathrm{TiO}_{2}$ retains the vibrational progression in the adsorbed states but with the $0-1$ transition becoming stronger than the $0-0$ transition. The trend observed in the absorption spectra, i.e. the different degrees of broadening, for the surface attached case follows the intuitive expectation based on the molecular structure of the different bridgeanchor groups (see Fig. 4).

As indicated in Fig. 7, the solvent spectra as well as those for the case of perylene attached to $\mathrm{TiO}_{2}$ could be rather well reproduced what gave the basis to fix all parameters as presented in Table I and II. Notice also that the replacement of $\overline{\mathcal{N}}$ by a frequency dependent DOS does not change the spectra (cf. Ref. [29]), indicating the validity of the wide-band approximation at the present mid-band position of the injection level.

\section{B. Charge Injection Near the Band Edge}

The results of the foregoing section are confronted in Fig. 8 with the absorption spectra one obtains if the charge injection would take place near the lower band-edge. Since no other data are available at the moment we again use the parameters derived for the perylene- $\mathrm{TiO}_{2}$ system with the only exception that the injection position $\hbar \varepsilon_{e}$ has been moved towards the lower conduction band edge. Moreover, a common frequency-dependent DOS

$$
\mathcal{N}(\Omega)=\nu \sqrt{\hbar \Omega-\hbar \omega_{\text {con }}}
$$

has been used (normalized to $\overline{\mathcal{N}}=2 / \mathrm{eV}$ in the energy interval from the band edge up to $1 \mathrm{eV}$ higher, cf. [29]). The spectra display the increasing resolution of the vibrational progression when moving with the injection position $\hbar \varepsilon_{e}$ near and below the band-edge. In any case the maximum of the absorbance is more than $0.1 \mathrm{eV}$ below the respective value of $\hbar \varepsilon_{e}$ indicating the effect of the band continuum induced shift of the excited electron-vibrational molecular levels (see the subsequent section). 


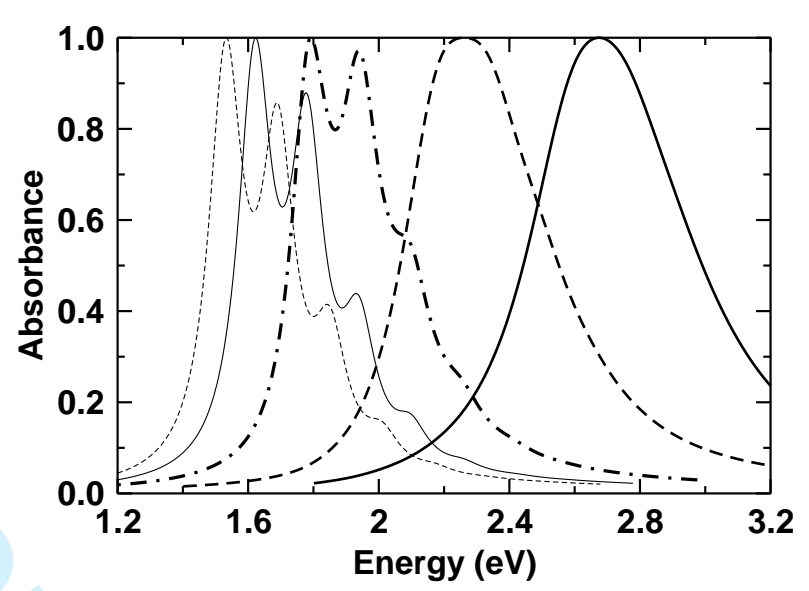

FIG. 8: Rescaled linear absorption spectra of a fictitious molecule at the $\mathrm{TiO}_{2}$ surface that can change the injection position $\hbar \varepsilon_{e}$. Solid line: $\hbar \varepsilon_{e}=2.79 \mathrm{eV}$ (corresponding to DTB-Pe-COOH), dashed line: $\hbar \varepsilon_{e}=2.39 \mathrm{eV}$, dashed-dotted line: $\hbar \varepsilon_{e}=2.19 \mathrm{eV}$, dotted line: $\hbar \varepsilon_{e}=1.99 \mathrm{eV}$, thin solid line: $\hbar \varepsilon_{e}=1.79 \mathrm{eV}$, thin dashed line: $\hbar \varepsilon_{e}=1.69 \mathrm{eV}$ (for all other parameters see Table I).

\section{Frequency Domain Description}

We change to the direct computation of the absorption coefficient in the frequencydomain. This will offer a simple picture of the influence of the excited molecular state conduction band coupling which is rather hidden in the time-dependent description. We make use of the Green's operator technique introduced in Section V and explained in detail in Appendix A. Noting Eq. (28) for the dipole-dipole correlation function and carrying out an expansion of $\hat{R}_{g}$ with respect to the vibrational eigenstates $\chi_{g N}$ what results in $\hat{R}_{g}=$ $\sum_{N} f\left(\hbar \omega_{g N}\right)\left|\chi_{g N}\right\rangle\left\langle\chi_{g N}\right|$ ( $f$ is the respective thermal distribution), we obtain

$$
\begin{aligned}
& \operatorname{tr}_{\mathrm{vib}}\left\{\hat{G}_{e e}(t) \hat{R}_{g} e^{i H_{g} t / \hbar}\right\}=\sum_{N} f\left(\hbar \omega_{g N}\right) e^{i \omega_{g N} t} \\
& \times \sum_{K, L}\left\langle\chi_{g N} \mid \chi_{e K}\right\rangle G_{e K, e L}(t)\left\langle\chi_{e L} \mid \chi_{g N}\right\rangle .
\end{aligned}
$$


The matrix elements $G_{e K, e L}$ have to be computed according to Eq. (A18). Of course this scheme is useful only in such situations where only a selected number of vibrational coordinates has to be considered.

In the wide-band approximation, Eq. (34) is reduced to (cf. Eq. (A20)):

$$
\begin{aligned}
& \alpha(\omega)=\frac{4 \pi \omega n_{\mathrm{mol}}\left|\mathbf{d}_{e g}\right|^{2}}{3 \hbar c} \sum_{N, K} f\left(\hbar \omega_{g N}\right)\left|\left\langle\chi_{g N} \mid \chi_{e K}\right\rangle\right|^{2} \\
& \times \frac{|\operatorname{Im} \bar{\Sigma}|}{\left(\omega-\left[\varepsilon_{e}+\omega_{e K}-\varepsilon_{g}-\omega_{g N}\right]-\operatorname{Re} \bar{\Sigma}\right)^{2}+(\operatorname{Im} \bar{\Sigma})^{2}} .
\end{aligned}
$$

The absorbance follows as an expression with Lorentzian line-shapes for every ground-state excited-state transition. The broadening is originated by the imaginary part of the selfenergy (cf. Eqs. (21) and (22)), whereas the real part of the self-energy induces a shift of the transition frequencies and reads $(\mathcal{P}$ indicates that the principle part of the integral has to be taken)

$$
\operatorname{Re} \Sigma(\omega)=\frac{1}{\hbar^{2}} \int d \Omega \mathcal{P} \frac{\mathcal{N}(\Omega)\left|V_{e}(\Omega)\right|^{2}}{\omega-\Omega}
$$

The frequency independent quantity $\operatorname{Re} \bar{\Sigma}$ appearing in Eq. (35) is obtained in replacing $\omega$ by a fixed frequency $\omega_{0}$, for example the actual transition frequency $\varepsilon_{e}+\omega_{e K}-\varepsilon_{g}-\omega_{g N}$. Once, $\omega_{0}$ is positioned around the lower conduction band edge $\omega_{\text {con }}$ the integrand is mainly negative and we expect a negative value of $\operatorname{Re} \bar{\Sigma}$. This negative shift of the transition frequencies becomes smaller when moving $\omega_{0}$ into a mid-band position (positive contributions to the overall $\Omega$-integral increase). Such a behavior is confirmed by our numerical calculations based on the time-domain formulation of the absorbance (see the discussion below).

The time-dependent formulation of the absorbance as displayed in Fig. 7 is compared with the formulation in the frequency-domain according to Eq. (35). Therefore, we concentrate on the strong-coupling case (upper panel of Fig. 7). The used values of $\bar{\Gamma} \equiv|\operatorname{Im} \bar{\Sigma}|$ 
are taken from Table I. To achieve complete agreement, however,a transition frequency shift originated by $\hbar \operatorname{Re} \bar{\Sigma}$ of about $-0.05 \mathrm{eV}$ has to be introduced. Interestingly, the combined effect of strong line broadening and a red shift of the transition frequencies gives the impression that the absorbance peak for the case of perylene attached to $\mathrm{TiO}_{2}$ stays at the same position as that for perylene in a solvent.

Finally, let us estimate the effect of structural and energetic disorder presumably present in all measured spectra. The easiest approach here to do this is the use of Eq. (35) and a restriction to fluctuations of the excited molecular level only. Of course, fluctuations of the molecular orientation at the surface might be possible (leading to fluctuations of the transfer coupling) and the surface structure of the semiconductor around the molecular binding site might also vary. But concentrating on the most simple case of molecular on-site disorder the respective disorder averaged absorbance follows by integrating Eq. (35) with respect to the disorder distribution of $\varepsilon_{e}$. This indicates, obviously, that the absorption spectra of Fig. (7) may be affected by inhomogeneous broadening. Then, the presented values of $\bar{\Gamma}$, Eq. (24), and of the rate $k_{\text {HET }}$ of HET for the perylene $\mathrm{TiO}_{2}$ systems (Tables I and II) are somewhat too large. Noting, however, the data in Table III the differences between $1 / k_{\mathrm{HET}}$ and the injection times measured via transient absorption data which are less effected by disorder may be diminished.

\section{Contributions of Charge Transfer States}

In order to be complete we next consider charge transfer state contributions to the absorbance. Here, however, the discussion stays on a rather general level without the presentation of any numerical results. To move forward we notice Eq. (7) which defines the respective part of the overall dipole operator (note that it represents an additional assumption that the intra-molecular excitation and the charge transfer state contribution are originated by the same ground-state of the molecule [39]). In similarity to Eq. (27) we obtain for the dipole-dipole correlation function (note that $a$ and $b$ cover $e$ and $\mathbf{k}$ )

$$
\begin{aligned}
& C_{\mathrm{d}-\mathrm{d}}(t)=\frac{1}{3} \sum_{a, b} \mathbf{d}_{a g}^{*} \mathbf{d}_{b g} \\
& \operatorname{tr}_{\mathrm{vib}}\left\{\left\langle\varphi_{a}\left|e^{-i H_{\mathrm{mol}-\mathrm{sem}} t / \hbar} \hat{R}_{g}\right| \varphi_{b}\right\rangle\left\langle\varphi_{g}\left|e^{i H_{\text {mol-sem }} t / \hbar}\right| \varphi_{g}\right\rangle\right\} .
\end{aligned}
$$


After introducing Green's operator electronic matrix elements the trace expression is expanded with respect to the vibrational states $\chi_{g M}$ and $\chi_{a M}(a=e, \mathbf{k})$. It yields:

$$
\begin{aligned}
& e^{i \varepsilon_{g} t} \operatorname{tr}_{\mathrm{vib}}\left\{\hat{G}_{a b}(t) \hat{R}_{g} e^{i H_{g} t / \hbar}\right\} \\
& =\sum_{M} f\left(\hbar \omega_{g M}\right) e^{i\left(\varepsilon_{g}+\omega_{g M}\right) t}\left\langle\chi_{g M}\left|\hat{G}_{a b}(t)\right| \chi_{g M}\right\rangle \\
& =\sum_{M, K, L} f\left(\hbar \omega_{g M}\right) e^{i\left(\varepsilon_{g}+\omega_{g M}\right) t} \\
& \times\left\langle\chi_{g M} \mid \chi_{a K}\right\rangle G_{a K, b L}(t)\left\langle\chi_{b L} \mid \chi_{g M}\right\rangle .
\end{aligned}
$$

If inserted into Eq. (25) for the absorbance we obtain

$$
\begin{aligned}
& \alpha(\omega)=-\frac{4 \pi \omega n_{\mathrm{mol}}}{3 c \hbar} \\
& \operatorname{Im}\left(\sum_{M, K, L} f\left(\hbar \omega_{g M}\right)\left\langle\chi_{g M} \mid \chi_{e K}\right\rangle\left\langle\chi_{e L} \mid \chi_{g M}\right\rangle\right. \\
& \mathbf{d}_{e g}^{*} \mathbf{d}_{e g} G_{e K, e L}\left(\omega+\varepsilon_{g}+\omega_{g M}\right) \\
& +\sum_{M, K, L} f\left(\hbar \omega_{g M}\right)\left\langle\chi_{g M} \mid \chi_{\mathrm{ion} K}\right\rangle\left\langle\chi_{e L} \mid \chi_{g M}\right\rangle \\
& \sum_{\mathbf{k}} \mathbf{d}_{\mathbf{k} g}^{*} \mathbf{d}_{e g} G_{\mathbf{k} K, e L}\left(\omega+\varepsilon_{g}+\omega_{g M}\right) \\
& +\sum_{M, K, L} f\left(\hbar \omega_{g M}\right)\left\langle\chi_{g M} \mid \chi_{e K}\right\rangle\left\langle\chi_{\mathrm{ion} L} \mid \chi_{g M}\right\rangle \\
& \sum_{\mathbf{q}} \mathbf{d}_{e g}^{*} \mathbf{d}_{\mathbf{q} g} G_{e K, \mathbf{q} L}\left(\omega+\varepsilon_{g}+\omega_{g M}\right) \\
& +\sum_{M, K, L} f\left(\hbar \omega_{g M}\right)\left\langle\chi_{g M} \mid \chi_{\mathrm{ion} K}\right\rangle\left\langle\chi_{\mathrm{ion} L} \mid \chi_{g M}\right\rangle \\
& \left.\sum_{\mathbf{k}, \mathbf{q}} \mathbf{d}_{\mathbf{k} g}^{*} \mathbf{d}_{\mathbf{q} g} G_{\mathbf{k} K, \mathbf{q} L}\left(\omega+\varepsilon_{g}+\omega_{g M}\right)\right) .
\end{aligned}
$$

The first term proportional to $\mathbf{d}_{e g}^{*} \mathbf{d}_{e g}$ reproduces the absorption coefficient according to Eq. (34) where only the intra-molecular excitation has been accounted for. In contrast the fourth term proportional to $\mathbf{d}_{\mathbf{k} g}^{*} \mathbf{d}_{\mathbf{q} g}$ generates the absorbance exclusively determined by direct charge transitions into the semiconductor band. The mixing of both transitions is included in the second and the third term. In particular, Eq. (39) indicates that the contribution of the intra-molecular transitions discussed in the foregoing sections is superimposed by an additional broad band. 


\section{TWO-PHOTON PHOTON EMISSION SPECTRA}

After having discussed frequency domain spectra we shortly indicate in the following how to calculate a particular type of transient spectra. Transient spectra may be related to an optical transition from the cationic ground state into an excited cation state. But also transitions addressing other product state are of interest. For example, such transitions may lift the injected electrons from the different states at the surface of the semiconductor into quasi-free electronic states above the vacuum level. There, the corresponding kinetic energy distribution of the emitted electrons is detected.

This type of pump-probe measurement is labeled as two-photon photo emission process and has been used as a method to characterize the temporal evolution of the injected electron in the semiconductor. Here, we present a preliminary description based on the injection dynamics studied in Section IV. The description includes a complete account of the laserpulse with field-strength $\mathbf{E}_{1}$ initiating charge injection in the time-dependent Schrödinger equation. In contrast, the photo emission caused by the second laser-pulse with field-strength $\mathbf{E}_{2}$ will be described in perturbation theory. Accordingly, the state of the system corresponding to the action of $\mathbf{E}_{2}$ can be written as (see, for example [5]):

$$
\left|\Psi^{(1)}(t)\right\rangle=\frac{i}{\hbar} \int_{t_{0}}^{t} d \bar{t} U\left(t, \bar{t} ; \mathbf{E}_{1}\right) \hat{\mu} \mathbf{E}_{2}(\bar{t})\left|\Psi\left(\bar{t} ; \mathbf{E}_{1}\right)\right\rangle .
$$

This is a first-order perturbation theory expression with respect to the photo emission process but it accounts in all orders for the action of the first pulse indicated by a dependence of the wave function $\Psi$ and the time-evolution operator $U$ on $\mathbf{E}_{1}$. The calculation of $\Psi$ has been described in Section IV. Here, we concentrate on an analysis of $\Psi^{(1)}$, Eq. (40). First we assume non-overlapping pulses, i.e. $U$ can be replaced by the field-independent expression $\exp \left(-i H_{\mathrm{mol}-\mathrm{sem}}(t-\bar{t}) / \hbar\right)$. Moreover, we neglect direct molecular contributions and expand $\Psi^{(1)}$ with respect to the states $\phi_{\kappa}^{(-)}$characterizing the freely moving electron (cf. Section III). It follows

$$
\chi_{\kappa}(t)=\frac{i}{\hbar} \int_{t_{0}}^{t} d \bar{t} e^{i \varepsilon_{\kappa}(t-\bar{t})} \mathbf{E}_{2}(\bar{t})\left\langle\phi_{\kappa}^{(-)}|\hat{\mu}| \Psi\left(\bar{t} ; \mathbf{E}_{1}\right)\right\rangle .
$$

The function $\chi_{\kappa}(t)$ is obtained as $\left\langle\phi_{\kappa}^{(-)} \mid \Psi^{(1)}\left(t ; \mathbf{E}_{1}\right)\right\rangle$ and describes the distribution of the emitted electron versus the quasi-free states $\phi_{\kappa}^{(-)}$(with energies $\hbar \varepsilon_{\kappa}$ ) and the vibrational state of the molecular cation. 
A detailed description of the charge injected state $\Psi$ can be achieved with the tightbinding model, i.e. using the atomic orbital basis for the semiconductor part. As a first estimate here we take the states $\varphi_{\mathbf{k}}$ and also assume an impulse excitation $\left(\mathbf{E}_{2}(\bar{t})=\right.$ $\mathbf{E}_{2}\left(t_{2}\right) \tau_{2} \delta\left(\bar{t}-t_{2}\right)$ with the pulse duration $\left.\tau_{2}\right)$ :

$$
\begin{aligned}
& \chi_{\kappa}(t)=\frac{i}{\hbar} \Theta\left(t-t_{2}\right) e^{i \varepsilon_{\kappa}\left(t-t_{2}\right)} \mathbf{E}_{2}\left(t_{2}\right) \tau_{2} \\
& \times \sum_{\mathbf{k} M}\left\langle\phi_{\kappa}^{(-)}|\hat{\mu}| \varphi_{\mathbf{k}}\right\rangle C_{\mathbf{k} M}\left(t_{2}\right) \chi_{\mathrm{ion} M} .
\end{aligned}
$$

If we further use simple plane waves for the description of the emitted electron and assume $\left\langle\phi_{\kappa}^{(-)}|\hat{\mu}| \varphi_{\mathbf{k}}\right\rangle \sim \delta_{\kappa, \mathbf{k}}$ the overall free electron distribution becomes

$$
P_{\mathbf{k}}^{(\mathrm{vac})}=\left\langle\chi_{\kappa}(t) \mid \chi_{\kappa}(t)\right\rangle \sim \sum_{M}\left|C_{\mathbf{k} M}\left(t_{2}\right)\right|^{2}
$$

We note Eq. (17) an write

$$
P^{(\mathrm{vac})}=\sum_{\mathbf{k}} P_{\mathbf{k}}^{(\mathrm{vac})} \sim P_{\mathrm{ion}}(t)
$$

If dispersed with respect to energy $\hbar \Omega$ we may conclude that $P^{(\mathrm{vac})}(\Omega)$ is proportional to the energetic distribution $P_{\mathrm{el}}\left(\Omega ; t_{2}\right)$, Eq. (18) of the injected electron versus the band states times the $\operatorname{DOS} \mathcal{N}(\Omega)$ already drawn in Fig. 6. Notice in this connection that vibrational signatures become observable although the electron transfer proceeds on a time-scale even below 10 fs.

\section{FEMTOSECOND LASER PULSE CONTROL}

Laser pulse guided molecular dynamics within closed loop control experiments represents one frontier in ultrafast optical spectroscopy (for a recent overview see [40, 41]). The whole research field is based on the vision to tailor femtosecond laser pulses in the optical and infrared region in order to drive the molecular wave function in a desired way. For the case of HET we discussed in Ref. [27] the control of the vibrational motion in the excited molecular state. However, the strong coupling of this level to the semiconductor band continuum suppressed any efficient manipulation of the vibrational motion. More efficient has been the control of the electronic ground-state vibrational states.

For the control to be discussed here it suffices to use a scheme of laser pulse control of molecular dynamics where one asks to realize a certain state $\left|\Psi_{\text {tar }}\right\rangle$ (the target state) at time 
$t_{f}$ (final time of laser pulse action) [42]. As it is well-known the laser pulse which solves this type of control task (the optimal pulse) can be derived from the extremum of the following functional (cf. e.g. $[5,43])$

$$
J\left(t_{f} ; \mathbf{E}\right)=\left|\left\langle\Psi_{\mathrm{tar}} \mid \Psi\left(t_{f}\right)\right\rangle\right|^{2}-\frac{1}{2} \int_{t_{0}}^{t_{f}} d t \lambda(t) \mathbf{E}^{2}(t) .
$$

This functional tries to realize a maximal overlap of the laser-pulse driven wavefunction $\left|\Psi\left(t_{f}\right)\right\rangle$ at time $t_{f}$ with the target state under the constraint of a finite field-strength of the pulse. In order to determine the extremum of $J\left(t_{f} ; \mathbf{E}\right)$ one fixes the penalty factor $\lambda$ instead of the whole field-pulse intensity. Therefore, it is only possible to establish a relationship between the intensity and $\lambda$ after the control problem has been solved. But Eq. (45) indicates that an increase of $\lambda$ should result in a decrease of the pulse intensity. The time-dependence of the Lagrangian multiplier $\lambda(t)=\lambda_{0} \sin ^{-2}\left(\pi t / t_{f}\right)$ has been introduced to realize a smooth switch on and switch off of the pulse at time $t_{0}$ and $t_{f}$, respectively.

The optimal pulse is obtained as the solution of the following functional equation (resulting from $\delta J=0)$

$$
\mathbf{E}(t)=-\frac{2}{\hbar \lambda(t)} \operatorname{Im}\left\{\left\langle\Psi\left(t_{f}\right) \mid \Psi_{\operatorname{tar}}\right\rangle\langle\Theta(t)|\hat{\mu}| \Psi(t)\rangle\right\}
$$

While $|\Psi(t)\rangle$ is obtained by the propagation of the Schrödinger equation starting with the initial value $\left|\Psi_{0}\right\rangle$, the state vector $|\Theta(t)\rangle$ follows from a backward propagation starting at $t_{f}$ with the "initial" value $\left|\Psi_{\text {tar }}\right\rangle$. The notation of the functional equation for the optimal pulse via a forward and a backward wavefunction propagation allows to formulate an efficient iteration scheme $[44,45]$.

Fig. 9 displays the results of a control task where the target state $\Psi_{\text {tar }}$ is given by the vibrational ground-state $\chi_{g 0}$ in the electronic ground-state but shifted away from its equilibrium position $\left(Q_{\text {shift }}=-1\right.$, for more details see Ref. [27]). Therefore, the control pulse introduces an excitation into the excited electronic level and back into the ground state (pump-dump scheme of laser pulse control, middle panel of Fig. 9). Since excitation and deexcitation covers a 20 fs interval the coupling to the continuum does not restricts the overall control yield (upper panel of Fig. 9). The dependence on the injection position, however, indicates that the control is more efficient for a near band-edge injection position. 

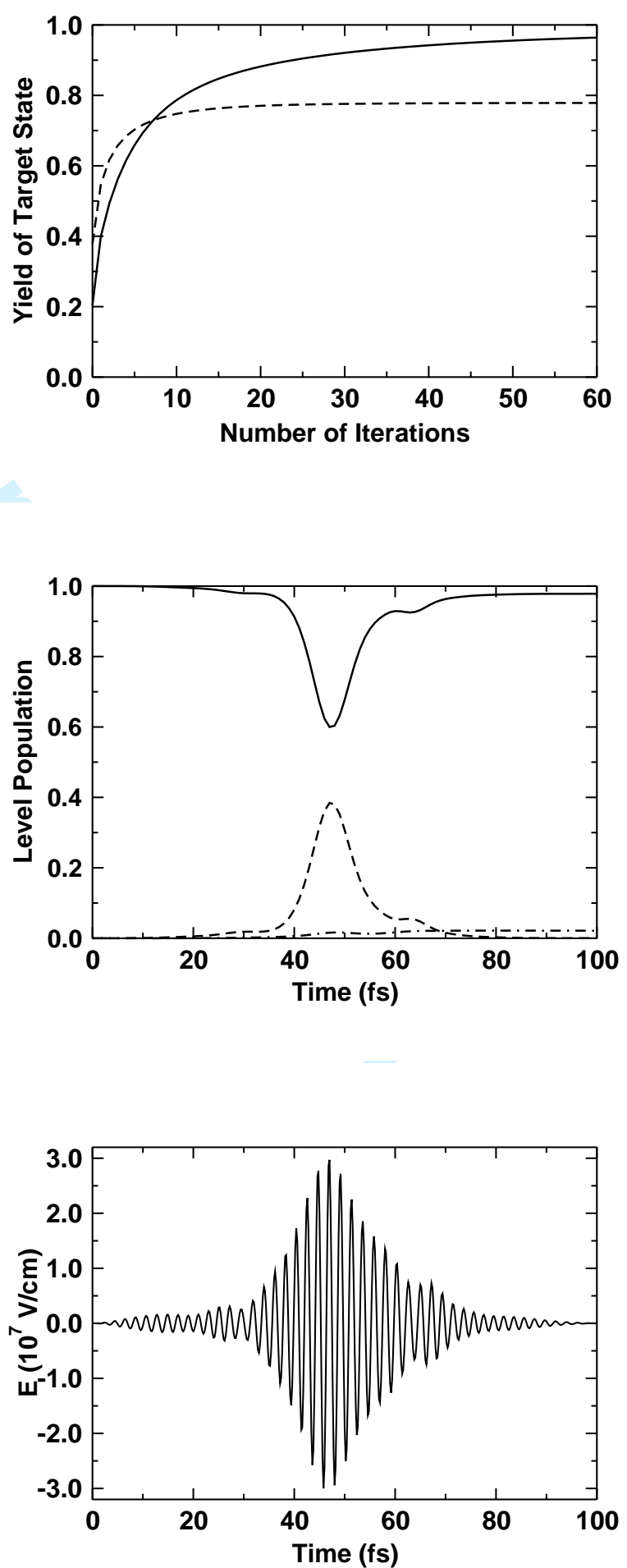

FIG. 9: Laser pulse control of the vibrational motion in the electronic ground-state. Variation of the injection position $\hbar \varepsilon_{e}$ into the band continuum. Upper panel: control efficiency versus the number of iteration steps, solid line: $\hbar \varepsilon_{e}=\hbar \varepsilon_{\text {con }}$ (band-edge injection), dashed line: $\hbar\left(\varepsilon_{e}-\varepsilon_{\text {con }}\right)=$ $1 \mathrm{eV}$. Middle panel: level population $P_{g}$ (solid line), $P_{e}$ (dashed line) and $P_{\text {ion }}$ (dashed-dotted line) versus time for the band-edge injection position. Lower panel: temporal behavior of the optimal pulse for the band-edge injection position. 


\section{CONCLUSIONS}

The present paper gives a survey of our recent theoretical studies on ultrafast heterogeneous electron transfer (HET) with emphasis on perylene on nano-structured $\mathrm{TiO}_{2}$ and also introduces some extensions. The approach accounts for molecular degrees of freedom as well as the band continuum of the semiconductor and allows to describe different spectroscopic excitation and detection processes. Such a uniform description of initiation, progression, and detection of HET required the use of a reduced dimensionality model. Since the studied HET proceeds on a femtosecond time-scale the respective time-dependent Schrödinger equation governing the electron-vibrational wave function is solved. All parameters of the used model could be specified by a comparison with measured steady state absorption spectra. Some preliminary considerations have been also presented on how to relate the energetic distribution of the injected electron to two-photon photon emission signals. In this connection we underline the possible observation of vibrational signatures in the two-photon photon emission signal although the electron transfer proceeds on a $10 \mathrm{fs}$ time-scale. Some speculations on femtosecond laser pulse control of the injection process have been given finally.

Although we consider the achieved description of ultrafast HET as rather complete there are different routes for further investigations. Of course, the used model might be extended in different respects, for example, by including further intra-molecular vibrational modes. However, there are no direct hints for an improved understanding of existing experimental data when doing this. More important would be an extension of the present studies to a picosecond time-scale. It requires the inclusion of different dissipation channels in particular the consideration of electron relaxation versus the conduction band states via electronphonon scattering. As an additional challenge we consider the further theoretical elaboration of two-photon photon emission processes at surfaces. Respective studies are in progress.

\section{Acknowledgments}

The financial support by the Deutsche Forschungsgemeinschaft through Sonderforschungsbereich 450 is gratefully acknowledged. 


\section{APPENDIX A: THE MOLECULE-SEMICONDUCTOR GREEN'S OPERATORS AND THEIR MATRIX ELEMENTS}

The Greenś operator corresponding to the overall time-evolution operator (without the field part) has been introduced in Eq. (19). It obeys the following equation of motion:

$$
i \hbar \frac{\partial}{\partial t} \hat{G}(t)=\hbar \delta(t)+H_{\mathrm{mol}-\mathrm{sem}} \hat{G}(t) .
$$

For further computations it is advisable to carry out a Fourier-transformation

$$
\hat{G}(\omega)=\int d t e^{i \omega t} \hat{G}(t)
$$

resulting in

$$
\left(\omega-H_{\mathrm{mol}-\mathrm{sem}} / \hbar\right) \hat{G}(\omega)=1 .
$$

To distinguish between the molecular levels $a=g, e$ and the conduction band states we introduce the projection operators

$$
\hat{\Pi}_{a}=\left|\varphi_{a}\right\rangle\left\langle\varphi_{a}\right|
$$

and

$$
\hat{\Pi}_{\mathrm{sem}}=\sum_{\mathbf{k}}\left|\varphi_{\mathbf{k}}\right\rangle\left\langle\varphi_{\mathbf{k}}\right|
$$

They obey

$$
\hat{\Pi}_{g}+\hat{\Pi}_{e}+\hat{\Pi}_{\mathrm{sem}}=1
$$

We define (the contribution of the molecular ground-state is of no interest) $\hat{G}_{e, e}(t)=$ $\hat{\Pi}_{e} \hat{G}(t) \hat{\Pi}_{e}, \quad \hat{G}_{e, \mathrm{sem}}(t)=\hat{\Pi}_{e} \hat{G}(t) \hat{\Pi}_{\mathrm{sem}}, \quad \hat{G}_{\mathrm{sem}, e}(t)=\hat{\Pi}_{\mathrm{sem}} \hat{G}(t) \hat{\Pi}_{e}$ and $\quad \hat{G}_{\mathrm{sem}, \mathrm{sem}}(t)=$ $\hat{\Pi}_{\text {sem }} \hat{G}(t) \hat{\Pi}_{\text {sem }}$, what will be used in the computations below.

\section{Computation of the Molecular Green's Operator}

We note Eq. (A3) and derive an equation for $\hat{G}_{e, e}$

$$
\hat{\Pi}_{e}\left(\omega-H_{\mathrm{mol}-\mathrm{sem}} / \hbar\right)\left(\hat{\Pi}_{g}+\hat{\Pi}_{e}+\hat{\Pi}_{\mathrm{sem}}\right) \hat{G}(\omega) \hat{\Pi}_{e}=\hat{\Pi}_{e},
$$

which can be written as (notice that $\hat{\Pi}_{g}$ does not contribute)

$$
\begin{aligned}
& \left(\omega-\varepsilon_{e}-H_{e} / \hbar\right) \hat{G}_{e, e}(\omega) \\
& -\hat{\Pi}_{e} H_{\mathrm{mol}-\mathrm{sem}} / \hbar \hat{\Pi}_{\mathrm{sem}} \hat{G}_{\mathrm{sem}, e}(\omega)=\hat{\Pi}_{e} .
\end{aligned}
$$


The quantity $\hat{G}_{\text {sem, } e}$ obeys

$$
\begin{aligned}
& 0=\hat{\Pi}_{\mathrm{sem}}\left(\omega-H_{\mathrm{mol}-\mathrm{sem}} / \hbar\right)\left(\hat{\Pi}_{g}+\hat{\Pi}_{e}+\hat{\Pi}_{\mathrm{sem}}\right) \hat{G}(\omega) \hat{\Pi}_{e} \\
& =-\hat{\Pi}_{\mathrm{sem}} H_{\mathrm{mol}-\mathrm{sem}} / \hbar \hat{\Pi}_{e} \hat{G}_{e, e}(\omega)+\left(\omega-H_{\mathrm{sem}} / \hbar\right) \\
& \times \hat{G}_{\mathrm{sem}, e}(\omega) .
\end{aligned}
$$

We introduced $H_{\text {sem }}=\hat{\Pi}_{\text {sem }} H_{\text {mol-sem }} \hat{\Pi}_{\text {sem }}$ and define

$$
\hat{G}_{\mathrm{sem}}^{(0)-1}(\omega)=\omega-H_{\mathrm{sem}} / \hbar
$$

It yields

$$
\hat{G}_{\mathrm{sem}, e}(\omega)=\hat{G}_{\mathrm{sem}}^{(0)}(\omega) \hat{\Pi}_{\mathrm{sem}} H_{\mathrm{mol}-\mathrm{sem}} / \hbar \hat{\Pi}_{e} \hat{G}_{e, e}(\omega)
$$

If inserted into the equation for $\hat{G}_{e, e}$ it follows

$$
\begin{aligned}
& \left(\omega-\varepsilon_{e}-H_{e} / \hbar-\hat{\Pi}_{e} H_{\mathrm{mol}-\mathrm{sem}} / \hbar \hat{\Pi}_{\mathrm{sem}}\right. \\
& \left.\times \hat{G}_{\mathrm{sem}}^{(0)}(\omega) \hat{\Pi}_{\mathrm{sem}} H_{\mathrm{mol}-\mathrm{sem}} / \hbar \hat{\Pi}_{e}\right) \hat{G}_{e, e}(\omega)=\hat{\Pi}_{e} .
\end{aligned}
$$

We analyze the extra term which depends on $H_{\text {mol-sem }}$ and get

$$
\begin{aligned}
& \hat{\Pi}_{e} H_{\mathrm{mol}-\mathrm{sem}} / \hbar \hat{\Pi}_{\mathrm{sem}} \hat{G}_{\mathrm{sem}}^{(0)}(\omega) \hat{\Pi}_{\mathrm{sem}} H_{\mathrm{mol}-\mathrm{sem}} / \hbar \hat{\Pi}_{e} \\
& =\frac{1}{\hbar^{2}} \sum_{\mathbf{k}} \frac{\left|V_{e, \mathbf{k}}\right|^{2}}{\omega-\varepsilon_{\mathbf{k}}-H_{\mathrm{ion}} / \hbar+i \epsilon} \hat{\Pi}_{e} \\
& \equiv \Sigma\left(\omega-H_{\mathrm{ion}} / \hbar\right) \hat{\Pi}_{e} .
\end{aligned}
$$

In the last part, the self-energy according to Eq. (21) has been used (note its dependence on $\left.H_{\text {ion }}\right)$. Its separation into the real an imaginary part results in

$$
\operatorname{Re} \Sigma(\omega)=\frac{1}{\hbar^{2}} \sum_{\mathbf{k}} \mathcal{P} \frac{\left|V_{e, \mathbf{k}}\right|^{2}}{\omega-\varepsilon_{\mathbf{k}}}
$$

and

$$
\operatorname{Im} \Sigma(\omega) \equiv-\Gamma(\omega)=-\frac{\pi}{\hbar^{2}} \sum_{\mathbf{k}}\left|V_{e, \mathbf{k}}\right|^{2} \delta\left(\omega-\varepsilon_{\mathbf{k}}\right) .
$$

Finally, this all gives the excited-state molecular Green's operator as

$$
\hat{G}_{e, e}(\omega)=\frac{\hat{\Pi}_{e}}{\omega-\varepsilon_{e}-H_{e} / \hbar-\Sigma\left(\omega-H_{\mathrm{ion}} / \hbar\right)+i \epsilon} .
$$




$$
\begin{aligned}
& \left(\omega-\varepsilon_{e}-\omega_{e K}\right) G_{e K, e L}(\omega) \\
& -\sum_{M}\left\langle\chi_{e K}\left|\Sigma\left(\omega-H_{\mathrm{ion}} / \hbar\right)\right| \chi_{e M}\right\rangle G_{e M, e L}(\omega)=\delta_{K, L},
\end{aligned}
$$

with the self-energy matrix elements

$$
\begin{aligned}
& \left\langle\chi_{e K}\left|\Sigma\left(\omega-H_{\text {ion }} / \hbar\right)\right| \chi_{e M}\right\rangle \\
& =\sum_{N}\left\langle\chi_{e K} \mid \chi_{\text {ion } N}\right\rangle \Sigma\left(\omega-\omega_{\text {ion } N}\right)\left\langle\chi_{\text {ion } N} \mid \chi_{e M}\right\rangle
\end{aligned}
$$

There are two limiting cases resulting in a simple expression for $G_{e K, e L}(\omega)$. First, let us assume that the reorganization energy for the charge injection is small. Then, we may conclude $\left\langle\chi_{\text {ion } N} \mid \chi_{e M}\right\rangle \approx \delta_{N, M}$, what results in $\left\langle\chi_{e K}\left|\Sigma\left(\omega-H_{\text {ion }} / \hbar\right)\right| \chi_{e M}\right\rangle \approx \delta_{K, M} \Sigma\left(\omega-\omega_{\text {ion } K}\right)$ and, thus, in

$$
G_{e K, e L}(\omega)=\frac{\delta_{K, L}}{\omega-\varepsilon_{e}-\omega_{e K}-\Sigma\left(\omega-\omega_{\text {ion } K}\right)+i \epsilon} .
$$

A similar expression is found in the framework of the so-called wide-band approximation where any frequency dependency of the self-energy is neglected. This leads to a formula equivalent to Eq. (A20) but with the replacement of $\Sigma\left(\omega-\omega_{\text {ion } K}\right)$ by the frequency independent expression $\bar{\Sigma}$.

\section{Computation of the Semiconductor Green's Operator}

We proceed similar to the foregoing section and get

$$
\begin{aligned}
& \hat{\Pi}_{\mathrm{sem}}\left(\omega-H_{\mathrm{mol}-\mathrm{sem}} / \hbar\right)\left(\hat{\Pi}_{g}+\hat{\Pi}_{e}+\hat{\Pi}_{\mathrm{sem}}\right) \\
& \times \hat{G}(\omega) \hat{\Pi}_{\mathrm{sem}}=\hat{\Pi}_{\mathrm{sem}}
\end{aligned}
$$


or

$$
\begin{aligned}
& \left(\omega-H_{\mathrm{sem}} / \hbar\right) \hat{G}_{\mathrm{sem}, \mathrm{sem}}(\omega)-\hat{\Pi}_{\mathrm{sem}} H_{\mathrm{mol}-\mathrm{sem}} / \hbar \hat{\Pi}_{e} \\
& \times \hat{G}_{e, \mathrm{sem}}(\omega)=\hat{\Pi}_{\mathrm{sem}} .
\end{aligned}
$$

The quantity $\hat{G}_{e \text {, sem }}$ obeys

$$
\begin{aligned}
& \hat{\Pi}_{e}\left(\omega-H_{\mathrm{mol}-\mathrm{sem}} / \hbar\right)\left(\hat{\Pi}_{g}+\hat{\Pi}_{e}+\hat{\Pi}_{\mathrm{sem}}\right) \hat{G}(\omega) \hat{\Pi}_{\mathrm{sem}} \\
& =\left(\omega-\varepsilon_{e}-H_{e} / \hbar\right) \hat{G}_{e, \mathrm{sem}}(\omega) \\
& -\hat{\Pi}_{e} H_{\mathrm{mol}-\mathrm{sem}} / \hbar \hat{\Pi}_{\mathrm{sem}} \hat{G}_{\mathrm{sem}, \mathrm{sem}}(\omega)=0 .
\end{aligned}
$$

We define

$$
\hat{G}_{e}^{(0)-1}(\omega)=\omega-\varepsilon_{e}-H_{e} / \hbar
$$

and get

$$
\hat{G}_{e, \operatorname{sem}}(\omega)=\hat{G}_{e}^{(0)}(\omega) \hat{\Pi}_{e} H_{\mathrm{mol}-\mathrm{sem}} / \hbar \hat{\Pi}_{\mathrm{sem}} \hat{G}_{\mathrm{sem}, \mathrm{sem}}(\omega) .
$$

If inserted into the equation for $\hat{G}_{\text {sem ,sem }}$ it follows

$$
\begin{aligned}
& \left(\omega-H_{\mathrm{sem}} / \hbar\right. \\
& \left.-\hat{\Pi}_{\mathrm{sem}} H_{\mathrm{mol}-\mathrm{sem}} / \hbar \hat{\Pi}_{e} \hat{G}_{e}^{(0)}(\omega) \hat{\Pi}_{e} H_{\mathrm{mol}-\mathrm{sem}} / \hbar \hat{\Pi}_{\mathrm{sem}}\right) \\
& \hat{G}_{\mathrm{sem}, \mathrm{sem}}(\omega)=\hat{\Pi}_{\mathrm{sem}} .
\end{aligned}
$$

We analyze the extra term which depends on $H_{\text {mol-sem }}$ and obtain

$$
\begin{aligned}
& \hat{\Pi}_{\mathrm{sem}} H_{\mathrm{mol}-\mathrm{sem}} / \hbar \hat{\Pi}_{e} \hat{G}_{e}^{(0)}(\omega) \hat{\Pi}_{e} H_{\mathrm{mol}-\mathrm{sem}} / \hbar \hat{\Pi}_{\mathrm{sem}} \\
& =\frac{1}{\hbar^{2}} \sum_{\mathbf{k}, \mathbf{q}} \frac{V_{\mathbf{k} e} V_{e \mathbf{q}}}{\omega-\varepsilon_{e}-H_{e} / \hbar+i \epsilon}\left|\varphi_{\mathbf{k}}\right\rangle\left\langle\varphi_{\mathbf{q}}\right| \\
& \equiv \hat{\Lambda}\left(\omega-H_{e} / \hbar\right)
\end{aligned}
$$

To compute vibrational state matrix elements we may deduce

$$
\begin{aligned}
& \left\langle\varphi_{\mathbf{k}} \chi_{\mathrm{ion} K}\right|\left(\omega-\varepsilon_{\mathbf{k}}-H_{\mathrm{ion}} / \hbar-\hat{\Lambda}\left(\omega-H_{e} / \hbar\right)\right) \\
& \times \hat{G}_{\mathrm{sem}, \operatorname{sem}}(\omega)\left|\varphi_{\mathbf{q}} \chi_{\mathrm{ion} L}\right\rangle=\delta_{\mathbf{k} K, \mathbf{q} L} .
\end{aligned}
$$

It results in

$$
\begin{aligned}
& \left(\omega-\varepsilon_{\mathbf{k}}-\omega_{\mathrm{ion} K}\right) G_{\mathbf{k} K, \mathbf{q} L}(\omega) \\
& -\sum_{\mathbf{p}, M}\left\langle\varphi_{\mathbf{k}} \chi_{\mathrm{ion} K}\left|\hat{\Lambda}\left(\omega-H_{e} / \hbar\right)\right| \varphi_{\mathbf{p}} \chi_{\mathrm{ion} M}\right\rangle G_{\mathbf{p} M, \mathbf{q} L}(\omega) \\
& =\delta_{\mathbf{k} K, \mathbf{q} L} .
\end{aligned}
$$


The $\Lambda$-operator matrix elements are obtained as

$$
\begin{aligned}
& \left\langle\varphi_{\mathbf{k}} \chi_{\mathrm{ion} K}\left|\hat{\Lambda}\left(\omega-H_{e} / \hbar\right)\right| \varphi_{\mathbf{p}} \chi_{\mathrm{ion} M}\right\rangle= \\
& \sum_{N}\left\langle\chi_{\mathrm{ion} K} \mid \chi_{e N}\right\rangle \frac{V_{\mathbf{k} e} V_{e \mathbf{p}}}{\omega-\varepsilon_{e}-\omega_{e N}+i \epsilon}\left\langle\chi_{e N} \mid \chi_{\mathrm{ion} M}\right\rangle \\
& \equiv V_{\mathbf{k} e} \lambda_{K M}(\omega) V_{e \mathbf{p}}
\end{aligned}
$$

with

$$
\lambda_{K M}(\omega)=\sum_{N} \frac{\left\langle\chi_{\mathrm{ion} K} \mid \chi_{e N}\right\rangle\left\langle\chi_{e N} \mid \chi_{\mathrm{ion} M}\right\rangle}{\omega-\varepsilon_{e}-\omega_{e N}+i \epsilon} .
$$

In the limit of small reorganization energy for the ground-state ionized-state transition one may deduce

$$
\lambda_{K M}(\omega) \approx \frac{\delta_{K, M}}{\omega-\varepsilon_{e}-\omega_{e K}+i \epsilon} .
$$

This all yields

$$
\begin{aligned}
& \left(\omega-\varepsilon_{\mathbf{k}}-\omega_{\mathrm{ion} K}\right) G_{\mathbf{k} K, \mathbf{q} L}(\omega) \\
& -\sum_{\mathbf{p}, M} V_{\mathbf{k} e} \lambda_{K M}(\omega) V_{e \mathbf{p}} G_{\mathbf{p} M, \mathbf{q} L}(\omega)=\delta_{\mathbf{k} K, \mathbf{q} L} .
\end{aligned}
$$

Finally we present mixed Green's operator matrix elements. We note Eq. (A11) and get

$$
\begin{aligned}
& \hat{G}_{\mathbf{k} K, e L}(\omega)=\left\langle\varphi_{\mathbf{k}} \chi_{\mathrm{ion} K}\right| \hat{G}_{\mathrm{sem}}^{(0)}(\omega) \hat{\Pi}_{\mathrm{sem}} H_{\mathrm{mol}-\mathrm{sem}} / \hbar \hat{\Pi}_{e} \\
& \times \hat{G}_{e}(\omega)\left|\varphi_{e} \chi_{e L}\right\rangle \\
& =\frac{V_{\mathbf{k} e} / \hbar}{\omega-\varepsilon_{\mathbf{k}}-\omega_{\mathrm{ion} K}+i \epsilon} \sum_{M}\left\langle\chi_{\mathrm{ion} K} \mid \chi_{e M}\right\rangle G_{e M, e L}(\omega) .
\end{aligned}
$$

In the same manner we may derive

$$
\begin{aligned}
& \hat{G}_{e K, \mathbf{q} L}(\omega)=\left\langle\varphi_{e} \chi_{e K}\right| \hat{G}_{e}^{(0)} \hat{\Pi}_{e} H_{\mathrm{mol}-\mathrm{sem}} / \hbar \hat{\Pi}_{\mathrm{sem}} \\
& \times \hat{G}_{\mathrm{sem}}\left|\varphi_{\mathbf{q}} \chi_{\mathrm{ion} L}\right\rangle \\
& =\sum_{\mathbf{p}, M} \frac{V_{e \mathbf{p}} / \hbar}{\omega-\varepsilon_{e}-\omega_{e K}+i \epsilon}\left\langle\chi_{e K} \mid \chi_{\mathrm{ion} M}\right\rangle G_{\mathbf{p} M, \mathbf{q} L}(\omega) .
\end{aligned}
$$


[1] D. DeVault, Quantum-Mechanical Tunneling in Biological Systems, 2nd ed. (Cambridge Univ. Press: London, 1984).

[2] E. G. Petrov, Physics of Charge Transfer in Biosystems (Naukowa Dumka: Kiev, 1984) (in Russian).

[3] R. A. Marcus and N. Sutin, Electron Transfer in Chemistry and Biology Biochim. Biophys. Acta 811, 265 (1985).

[4] J. Jortner and M. Bixon (eds.), Electron Transfer, Adv. Chem. Phys. 106, 107 (1999).

[5] V. May and O. Kühn, Charge and Energy Transfer Dynamics in Molecular Systems (WileyVCH, Berlin, 2004).

[6] V. May, M. Schreiber, Electron Transfer Dynamics in a Donor Acceptor Complex Chem. Phys. Lett. 181, 267 (1991).

[7] V. May, M. Schreiber, Density Matrix Theory of Charge Transfer Phys. Rev. A 45, 2868 (1992).

[8] J. M. Jean, R. A. Friesner, and G. R. Fleming, Application of a Multilevel Redfield Theory to Electron Transfer in Condensed Phases J. Chem. Phys.96, 5827 (1992).

[9] A. Kühl and W. Domcke, Multilevel Redfield Description of the Dissipative Dynamics at Conical Intersections, J. Chem. Phys. 116, 263 (2002).

[10] T. Mančal, U. Kleinekathöfer, and V. May, Femtosecond Laser Pulse Control of Electron Transfer, J. Chem. Phys. 117, 636 (2002).

[11] D. Egorova, M. Thoss, W. Domcke, and H. Wang, Modeling of Ultrafast Electron-Transfer Processes: Validity of Multilevel Redfield Theory, J. Chem. Phys. 119, 2761 (2003).

[12] H. Wang and M. Thoss, Theoretical Study of Ultrafast Photoinduced Electron Transfer Processes in Mixed-Valence Systems, J. Phys. Chem. A 107, 2126 (2003).

[13] The Spin-Boson Problem: From Electron Transfer to Quantum Computing, special issue, Chem. Phys. 296, 101 (2004).

[14] B. O'Regan, M. and Grätzel, A Low-Cost, High-Efficiency Solar Cell Based on Dye-Sensitized Colloidal $\mathrm{TiO}_{2}$ Films, Nature 353, 737 (1991).

[15] R. J. D. Miller, G. McLendon, A. Nozik, W. Schmickler, and F. Willig, Surface Electron 
Transfer Processes (VCH Publishers, New York, 1995).

[16] J. B. Asbury, E. Hao, T. Wang, H. N. Ghosh, and T. Lian, Ultrafast Electron Transfer Dynamics from Adsorbate Molecules to Nanocrystalline Semiconductor Thin Films, J. Phys. Chem. B 105, 4545 (2001).

[17] J. Kallioinen, G. Benkö, V. Sundström, J. E. I. Korppi-Tommola,and A. P. Yartsev, Electron Transfer from the Singlet and Triplet Excited States of Ru(dcbpy $)_{2}(\mathrm{NCS})_{2}$ into Nanocrystalline $\mathrm{TiO}_{2}$ Thin Films, J. Phys. Chem. B106, 4396 (2002).

[18] A. Hagfeldt, and M. Grätzel, Light-Induced Redox Reactions in Nanocrystalline Systems, Chem. Rev.95, 49 (1995).

[19] K. Schwarzburg, R. Ernstorfer, S. Felber, and F. Willig, Primary and Final Charge Separation in the Nano-Structured Dye-Sensitized Electrochemical Solar Cell, Coord. Chem. Rev. 248, $1259(2004)$.

[20] J. M. Rehm, G. L. McLendon, Y. Nagasawa, K. Yoshihara, J. E. Moser, M. Grätzel, and J. Wachveitel, Femtosecond Electron-Transfer Dynamics at a Sensitizing Dye-Semiconductor $\left(\mathrm{TiO}_{2}\right)$ Interface, J. Phys. Chem. 100, 9577 (1996).

[21] C. Zimmermann, F. Willig, S. Ramakrishna, B. Burfeindt, B. Pettinger, R. Eichberger, W. and Storck, Experimental Fingerprints of Vibrational Wave-Packet Motion during Ultrafast Heterogeneous Electron Transfer, J. Phys. Chem. B 105, 9245 (2001).

[22] R. Huber, J. E. Moser, M. Grätzel, and J. Wachveitel, Real-Time Observation of Photoinduced Adiabatic Elctron Transfer in Strongly Coupled Dye/Semiconductor Colloidal Systems with a 6 fs Time Constant, J. Phys. Chem. B 106, 6494, (2002).

[23] L. Gundlach, S. Felber, W. Storck, E. Galoppini, Q. Wei and, F. Willig, Femtosecond TwoPhoton Photoemission Probing Electron Injection from the Excited Singlet State of Perylene Attached to a Long Rigid Tripod Anchor-Cumspacer on Rutile $\mathrm{TiO}_{2}(110)$, Res. Chem. Intermed. 31, 39 (2005).

[24] S. Ramakrishna, F. Willig, and V. May, A Franck-Condon Dictated Electronic Wavepacket: Photoinduced Ultrafast Electron Injection from a Surface Attached Molecule, Phys. Rev. B 62, R16330 (2000).

[25] S. Ramakrishna, F. Willig, and V. May, Bridge Mediated Ultrafast Heterogeneous Electron Transfer, Chem. Phys. Lett. 351, 242 (2002).

[26] S. Ramakrishna, F. Willig, V. May, and A. Knorr, Femtosecond Spectroscopy of Heteroge- 
neous Electron Transfer: Extraction of Excited State Population Dynamics from Pump-Probe Signals, J. Phys. Chem. B 107, 607 (2003).

[27] L. Wang and V. May, Laser Pulse Control of Ultrafast Heterogeneous Electron Transfer: A Computational Study, J. Chem. Phys. 121, 8039 (2004).

[28] L. Wang, R. Ernstorfer, F. Willig, and V. May, Absorption Spectra Related to Heterogeneous Electron Transfer Reactions: The Perylene-TiO 2 System, J. Phys. Chem. B 109, 9589 (2005).

[29] L. Wang, F. Willig, and V. May, Ultrafast Heterogeneous Electron Transfer Reactions: Comparative Theoretical Studies in the Time and Frequency Domain, J. Phys. Chem. B 109, 9589 (2005).

[30] M. Thoss, I. Kondov, and H. Wang, Theoretical Study of Ultrafast Heterogeneous Elctron Transfer Reactions at Dye-Semiconductor Interface, Chem. Phys. 304, 169 (2004).

[31] I. Kondov, M. Thoss, and H. Wang, Theoretical Study of Ultrafast Heterogeneous Elctron Transfer Reactions at Dye-Semiconductor Interface: Coumarin 343 at Titanium Oxide, J. Phys. Chem. A 110, 1364 (2006).

[32] P. Persson, R. Bergström, and S. Lunell, Quantum Chemical Study of Photoinjection Processes in Dye-Sensitized $\mathrm{TiO}_{2}$ Nanoparticles, J. Phys. Chem. B 104, 10348 (2000).

[33] L. G. C. Rego and V. S. Batista, Quantum Dynmiacs Simulation of Interfacial Elctron Transfer in Sensitized $\mathrm{TiO}_{2}$ Semiconductors, J. Am. Chem. Soc. 125, 7989 (2003).

[34] W. R. Duncan, W. M. Stier, and O. V. Prezhdo, Ab initio Nonadiabatic Molecular Dynamics of the Ultrafast Electron Injection across the Alizarin-TiO ${ }_{2}$ Interface, J. Am. Chem. Soc. 127, 7941 (2005).

[35] P. Persson, M. J. Lundqvist, R. Ernstorfer, W. A. Goddar III, and F. Willig, Quantum Chemical Calculations of the Influence of Anchor-Cum-Spacer Groups on Femtosecond Electron Transfer Times in Dye-Sensitized Semiconductor Nanocrystals, J. Chem. Theory. Comput. 2, 441 (2006).

[36] T. M. Halasinski, J. L. Weisman, R. Ruiterkamp, T. J. Lee, F. Salama and M. HeadGordon,Electronic Absorption Spectra of Neutral Perylene $\left(\mathrm{C}_{2} \mathrm{OH}_{1} 2\right)$, Terrylene $\left(\mathrm{C}_{3} \mathrm{OH}_{1} 6\right)$, and Quaterrylene $\left(\mathrm{C}_{4} \mathrm{OH}_{2} \mathrm{O}\right)$ and their Positive and Negative Ions: Ne Matrix-Isolation Spectroscopy and Time Dependent Density Functional Theory Calculations, J. Phys. Chem. A 107, 3660 (2003).

[37] K. K. Ong, J. O. Jensen, and H. F. Hameka, Theoretical Studies of the Infrared and Raman 
Spectra of Perylene, J. Mol. Str. (Theochem), 459, 131 (1999).

[38] R. Ernstorfer, L. Gundlach, S. Felber, W. Storck, R. Eichberger, and F. Willig (to be published).

[39] L. Wang, F. Willig, and V. May (in preparation).

[40] J. L. Herek (ed.), Coherent Control of Photochemical and Photobiological Processes, special issue, J. Photochem. Photobiol. (2006).

[41] Th. Halfmann (ed.), Quantum Control of Light and Matter, special issue, Opt. Comm. (2006).

[42] A. P. Pierce, M. A. Dahleh, and H. Rabitz, Optimal Control of Quantum-Mechanical Systems: Existence, Numerical Approximation, and Applications, Phys. Rev. A 37, 4950 (1988).

[43] Y. J. Yan, R. E. Gillian, R. M. Whitnell, K. R. Wilson, and S. Mukamel, Optical Control of Molecular Dynamics: Liouville-Space Theory, J. Phys. Chem. 97, 2320 (1993).

[44] W. Zhu, J. Botina, and H. Rabitz, Rapidly Convergent Iteration Methods for Quantum Optimal Control of Population, J. Chem. Phys. 108, 1953 (1998).

[45] W. Zhu and H. Rabitz, A Rapid Monotonically Convergent Iteration Algorithm for Quantum Optimal Control over the Expectation Value of a Positive Definite Operator, J. Chem. Phys. 109, 385 (1998). 